\title{
A dynamical approach to the spatiotemporal aspects of the Portevin-Le Chatelier effect: Chaos, turbulence and band propagation
}

\author{
G. Ananthakrishna ${ }^{1,2}$ and M.S. Bharathi ${ }^{1}$ \\ ${ }^{1}$ Materials Research Centre, Indian Institute of Science, Bangalore-560012, India \\ 2 Centre for Condensed Matter Theory, Indian Institute of Science, Bangalore-560012, India
}

\begin{abstract}
The analysis of experimental time series obtained from single and poly-crystals subjected to a constant strain rate tests report an intriguing dynamical crossover from a low dimensional chaotic state at medium strain rates to an infinite dimensional power law state of stress drops at high strain rates. We present results of an extensive study of all aspects of the PLC effect within the context a recent model that reproduces this crossover. We characterize the dynamics of this crossover by studying the distribution of the Lyapunov exponents as a function of the strain rate, with special attention to system size effects. The distribution of the exponents changes from a small set of positive exponents in the chaotic regime to a dense set of null exponents in the scaling regime. As the latter is similar to the result in the GOY shell model for turbulence, we compare the results of our model with that of the GOY model. Interestingly, the null exponents in our model themselves obey a power law. The study is complimented by visualizing the configuration of dislocations through the slow manifold analysis. This shows that while a large proportion of dislocations are in the pinned state in the chaotic regime, most of them are pushed to the threshold of unpinning in the scaling regime, thus providing an insight into the mechanism of crossover. We also show that this model qualitatively reproduces the different types of deformation bands seen in experiments. At high strain rates where propagating bands are seen, the model equations can be reduced to the Fisher-Kolmogorov equation for propagative fronts. Marginal stability analysis shows that the velocity of the propagating of the bands varies linearly with the strain rate and inversely with the dislocation density. These results are consistent with the known experimental results. We also discuss the connection between the nature of band types and the dynamics in the respective regimes. The analysis demonstrates that this simple dynamical model captures the complex spatio-temporal features of the PLC effect.
\end{abstract}

PACS numbers: 05.65.+b, 05.45.Ac, 62.20.Fe, 05.90.+m

\section{INTRODUCTION}

Plastic deformation is a highly dissipative irreversible nonequilibrium process where nonlinearities play a fundamental role. Under normal conditions, one finds homogeneous deformation. However, under suitable conditions of deformation, different types of spatial and temporal patterns are observed. At a microscopic level, these patterns arise due to the collective behavior of dislocations. These can be broadly classified by the associated time and length scales. For example, the persistent slip bands observed in cyclic deformation is an example of nearly permanent pattern lasting over long time scales [1]. On the other hand, a type of propagative bands referred to as the Lüders bands observed in uniaxial tension tests are characterized by short time scale [1]. Yet another and even more complex spatio-temporal patterns are observed during tension tests of dilute metallic alloys in a certain range of strain rates and temperatures. This phenomenon has come to be known as the Portevin-Le Chatelier (PLC) effect [2]. Here a uniform deformation mode becomes unstable leading to a spatially and temporally inhomogeneous state. The instability manifests itself in the form of serrations on the stress-strain curves of the sample [1, 3]. Each stress drop is generally associated with the nucleation and often the propagation of a band of localized plastic deformation. In poly-crystals, these bands and the associated serrations are classified into three generic types. On increasing strain rate or decreasing the temperature, one first finds the type $\mathrm{C}$ band, identified with randomly nucleated static bands with large characteristic stress drops on the stress- strain curve. The serrations are quite regular. Then the type B 'hopping' bands are seen. The serrations are more irregular with amplitudes that are smaller than that for the type C. The bands formed are still localized and static in nature, but forming ahead of the previous band in a spatially correlated way giving the visual impression of a hopping propagation. Finally, one observes the continuously propagating type A bands associated with small stress drops. (In single crystals such a clear classification does not exist.) These different types of PLC bands are believed to represent distinct correlated states of dislocations in the bands.

The well accepted classical explanation of the PLC effect is via the dynamic strain aging (DSA) concept first introduced by Cottrell [4] and later extended by others [1, 5, 6, 7]. In the Cotrell's picture, the dynamic strain aging refers to the interaction of mobile dislocations with the diffusing solute atoms. At low strain rates (or high temperatures) the average velocity of dislocations is low and there is sufficient time for the solute atoms to diffuse to the dislocations and pin them (called as aging). Thus, longer the dislocations are arrested, larger will be the stress required to unpin them. When these dislocations are unpinned, they move at large speeds till they are arrested again. At high strain rates (or low temperatures), the time available for solute atoms to diffuse to the dislocations decreases and hence the stress required to unpin them decreases. Thus, in a range of strain rates and temperatures where these two time scales are of the same order of magnitude, the PLC instability manifests. The competition between the slow rate of pinning and sudden unpinning of the dislocations, at the macroscopic level translates into a negative strain rate sensitivity (SRS) 
of the flow stress as a function of strain rate which is the basic instability mechanism used in most phenomenological models [1, 3]. Slow-fast dynamics and the negative flow rate characteristic is common to many stick-slip systems such as frictional sliding [8], fault dynamics [9] and peeling of an adhesive tape [10] and charge density waves [11].

There are two different types of challenges in dealing with the PLC effect. First, understanding the collective behavior of dislocations which has been slow largely due to lack of techniques for describing the cooperative behavior of dislocations. Second, the PLC effect involves collective modes of dislocations where both fast and slow times scales play equally important role which requires specific techniques of nonlinear dynamics (as we shall see). Further, these time scales themselves evolve as a function of strain rate and temperature which in turn leads to different types of serrations. At low strain rate, the existence of both fast ( time scales over which stress drops occur) and slow time scales ( loading time scales) are clearly displayed in the stress -strain curves. However, at high strain rate, as internal (plastic) relaxation is not complete, and a clear demarkation of time scales becomes difficult. This along with the corresponding length scales ( band widths), which also evolve, points to an extremely complex underlying dynamics.

The inherent nonlinearity and the presence of multiple time scales necessitates the use of tools and concepts of nonlinear dynamics for a proper understanding of this phenomenon. Early theories based on DSA do not deal with the temporal aspect [5, 6, 7] and thus are unsuitable for analyzing the dynamical aspects of the PLC effect. The first dynamical approach was undertaken in early 80 s by Ananthakrishna and coworkers [12], which by its very nature affords a natural basis for the description of the time dependent aspects of the PLC effect. Further, it also allows for explicit inclusion and interplay of different time scales inherent in the dynamics of dislocations. The original model which attempts to address the time dependence of the phenomenon uses three types of dislocation densities assumed to represent the collective degrees of freedom of dislocations [12]. Despite the simplicity of the model, many generic features of the PLC effect such as the existence of a window of strain rates and temperatures within which it occurs, etc., were correctly reproduced. More importantly, the negative SRS was shown to emerge naturally in the model, as a result of nonlinear interaction of the participating defects [12, 13].

Due to the dynamical nature of the model, one prediction that is unique to this model is the existence of the chaotic stress drops in a certain range of temperatures and strain rates [14]. This triggered a series of experiments to verify this prediction. The method followed was to analyze the stress-time series [15, 16] using dynamical methods [17, 18]. Apart from confirming the chaotic nature of stress drops in a window of strain rates, these attempts have shown that a wealth of dynamical information can be extracted from the stress-time series obtained during the PLC effect [15, 16]. Indeed, the number of degrees of freedom estimated from the experimental time series turn out to be same as in the model offering justi- fication for ignoring spatial degrees of freedom. Subsequent efforts to extend this analysis to the time series obtained over a range of strain rates showed an intriguing crossover from a chaotic state at low and medium strain rates to a power law state at high strain rates [19, 20]. As the crossover is observed in both single and polycrystals, it appears to be insensitive to the microstructure. However, chaotic state is dynamically a distinct state from the power law state as the former involves a small number of degrees of freedom characterized by the selfsimilarity of the attractor and sensitivity to initial conditions [18] while latter is an infinite dimensional state reminiscent of self-organized criticality (SOC) [21, 22, 23]. Due to this basic difference in the nature of the dynamics, most systems exhibit either of these states. More importantly, these studies also demonstrate that the nature of the dynamics in a given strain regime is correlated with the nature of band type. The chaotic state has been identified with the type B bands and the scaling regime at high strain rate with the propagating type A bands [20]. These authors also make a connection between the transition in the nature of serration between the type B and type A bands regime of strain rates with the Anderson's transition in condensed matter physics. Indeed, recently the spatiotemporal features of the PLC effect have attracted attention from physicists also [24]. Thus, it appears that the PLC effect is a storehouse of many paradigms in condensed matter physics. Understanding these connections between dynamics and general features of the PLC effect would give insight into the rich physics. As the above studies underscore the importance of nonlinearity, it demands a dynamical approach to the PLC effect.

The dynamics of the crossover as a function of strain rate is unusual in a number of ways. First, the PLC effect is one of the two rare instances where such an intriguing crossover phenomenon is seen, the other being in the hydrodynamic turbulence [26]. Second, the power law, both in the PLC effect and turbulence, arises at high drive rates [26, 27]. Thus, it would be interesting to examine the similarity and differences with hydrodynamic turbulence by comparing results of the Lyapunov spectrum of the model for the PLC effect and GOY shell model of turbulence [27, 28]. Another motivation is that such a study helps us to compare the nature of the Lyapunov spectrum with the conventional SOC systems seen at low drives ( such as those in earthquakes [29], acoustic emission during volcanic activity [30], Barkhausen noise [31]). ( For lack of anything better, we shall reserve SOC for power law situations at low drives.) Finally, as different types of bands are a characteristic feature of the PLC effect, we investigate the connection between spatial aspects and the nature of the dynamics.

The fully dynamical nature of the model and it prediction of chaotic stress drops at intermediate strain rates as found in experiments, makes it most suitable for studying this crossover by including spatial degrees of freedom. The paper reports a detailed investigation of all these issues ( some of which has been reported in brief earlier [32, 33]) within the context of an extension of Ananthakrishna's model for the PLC effect. Particular attention will be paid to study the system size effects during the crossover. 
Section II, briefly introduces the dynamical model and its extension to include spatial degrees of freedom. Section III contains the numerical procedure used. In Section IV, we introduces the background material used for the study. Section $\mathrm{V}$ contains a comparison of the results of analysis of experimental time series with that of the model. Section VI, contains all the major results on the dynamics of crossover including the evolution of the Lyapunov spectrum with special attention to study the system size effects as a function of the strain rate along with the analysis of the distribution of null exponents in the power law regime of stress drops. The section also includes a comparison of the results of the model with that of the GOY model for turbulence followed by the slow manifold method of visual realization of dislocation configurations. Finally, in section VII we discuss both analytical and numerical results on the nature of dislocation bands. We conclude the paper with a few general comments.

\section{THE ANANTHAKRSHANA'S MODEL}

In the dynamical model due to Ananthakrishna and coworkers [12], the well separated time scales mentioned in the DSA are mimicked by three types of dislocations, namely, the fast mobile, immobile and the 'decorated' Cottrell type dislocations. The basic idea of the model is that all the qualitative features of the PLC effect emerge from the nonlinear interaction of these few dislocation populations, assumed to represent the collective degrees of freedom of the system. As the model has been studied in detail by our group and others including an extension to the case of fatigue [34, 35, 36], following the notation in Ref. [13], we shall briefly outline the model in the scaled variables. In our model, a natural basis for including the spatial coupling is through the cross-slip mechanism proposed earlier [1] with an important difference (see below). The model consists of densities of mobile, immobile, and Cottrell's type dislocations denoted by $\rho_{m}(x, t), \rho_{i m}(x, t)$ and $\rho_{c}(x, t)$ respectively, in the scaled form. The evolution equations are:

$$
\begin{aligned}
\frac{\partial \rho_{m}}{\partial t} & =-b_{0} \rho_{m}^{2}-\rho_{m} \rho_{i m}+\rho_{i m}-a \rho_{m}+\phi_{e f f}^{m} \rho_{m} \\
& +\frac{D}{\rho_{i m}} \frac{\partial^{2}\left(\phi_{e f f}^{m}(x) \rho_{m}\right)}{\partial x^{2}} \\
\frac{\partial \rho_{i m}}{\partial t} & =b_{0}\left(b_{0} \rho_{m}^{2}-\rho_{m} \rho_{i m}-\rho_{i m}+a \rho_{c}\right), \\
\frac{\partial \rho_{c}}{\partial t} & =c\left(\rho_{m}-\rho_{c}\right) .
\end{aligned}
$$

The model includes the following dislocation mechanisms: immobilization of two mobile dislocations due to the formation of locks $\left(b_{0} \rho_{m}^{2}\right)$, the annihilation of a mobile dislocation with an immobile one $\left(\rho_{m} \rho_{i m}\right)$, the remobilisation of the immobile dislocation due to stress or thermal activation $\left(\rho_{i m}\right)$. It also includes the immobilisation of mobile dislocations due to solute atoms $\left(a \rho_{m}\right)$. Once a mobile dislocation starts acquiring solute atoms we regard it as the Cottrell's type dislocation $\rho_{c}$.
As they progressively acquire more solute atoms, they eventually stop, then they are considered as immobile dislocations $\rho_{i m}$. Alternately, the aggregation of solute atoms can be regarded as the definition of $\rho_{c}$, ie., $\rho_{c}=\int_{-\infty}^{t} d t^{\prime} \rho_{m}\left(t^{\prime}\right) K\left(t-t^{\prime}\right)$, where $K(t)$ is an appropriate kernel. For the sake of simplicity, this kernel is modelled through a single time scale, $K(t)=e^{-c t}$ . The convoluted nature of the integral physically implies that the mobile dislocations to which solute atoms aggregate earlier will be aged more than those which acquire solute atoms later (see ref. [13]). The fifth term in Eqn.(1) represents the rate of multiplication of dislocations due to cross-slip. This depends on the velocity of the mobile dislocations taken to be $V_{m}(\phi)=\phi_{e f f}^{m}$, where $\phi_{e f f}=\left(\phi-h \rho_{i m}^{1 / 2}\right)$ is the scaled effective stress, $\phi$ the scaled stress, $m$ the velocity exponent and $h$ a work hardening parameter.

The nature of the spatial coupling in the PLC effect has been a matter of much debate [1]. Several mechanisms have been suggested as a source of spatial coupling, such as compatibility stresses between the slipped and the un-slipped regions, long range interactions, and triaxiality of stresses [1]. Within the scope of our model, cross-slip is a natural source of spatial coupling, as dislocations generated due to cross slip at a point spread over to the neighboring elements. Let $\Delta x$ be an elementary length. Then, the flux $\Phi(x)$ flowing from $x \pm \Delta x$ and out of $x$ is given by

$$
\left.\Phi(x)+\frac{p}{2}[\Phi(x+\Delta x)-2 \Phi(x)+\Phi(x-\Delta x)]\right) .
$$

where $\Phi(x)=\rho_{m}(x) V_{m}(x)$ and $p$ is the probability of crossslip spreading into neighboring elements. Expanding $\Phi(x \pm$ $\Delta x)$ and keeping the leading terms, we get

$$
\rho_{m} V_{m}+\frac{p}{2} \frac{\partial^{2}\left(\rho_{m} V_{m}\right)}{\partial x^{2}}(\Delta x)^{2} .
$$

We further note that cross-slip spreads only into regions of minimum back stress. Here, we consider the back stress is taken to result from the immobile dislocation density ahead of it. Thus, we use $\Delta x^{2}=\left\langle\Delta x^{2}\right\rangle=\bar{r}^{2} \rho_{i m}^{-1}$, where $<\ldots>$ refers to the ensemble average and $\bar{r}^{2}$ is an elementary (dimensionless) length. Finally, $a, b_{0}$ and $c$ are the scaled rate constants referring, respectively, to the concentration of solute atoms slowing down the mobile dislocations, the thermal and athermal reactivation of immobile dislocations, and the rate at which the solute atoms are gathering around the mobile dislocations. We note here that the order of magnitudes of the constants have been identified in Ref. [12, 35, 36]. These equations are coupled to the machine equation

$$
\frac{d \phi(t)}{d t}=d\left[\dot{\varepsilon}-\frac{1}{l} \int_{0}^{l} \rho_{m}(x, t) \phi_{e f f}^{m}(x, t) d x\right],
$$

where $\dot{\varepsilon}$ is the scaled applied strain rate, $d$ the scaled effective modulus of the machine and the sample, and $l$ the dimensionless length of the sample. (We reserve $\dot{\varepsilon}_{a}$ for the unscaled strain rate.) We also note here that there is a feed back mechanism between Eq. 6 and Eq.(1). The machine equation which determines the stress depends on the the difference between the applied strain rate and average plastic strain rate generated 
in the sample. Thus, the nature of internal relaxation can influence stress generated in the sample which in turn determines the dislocation multiplication in Eq. (1). This type of global coupling (Eq. 6) is common to many other situations for instance in the nonlinear transport properties of charge density waves ( in blue bronze for example) [11]. We shall make some comments on this later.

\section{NUMERICAL SOLUTION OF THE MODEL}

We first note that the spatial dependence of $\rho_{\text {im }}$ and $\rho_{c}$ arises only through that of $\rho_{m}$. We solve the above set of equations by discretizing the specimen length into $N$ equal parts. Then, $\rho_{m}(j, t), \rho_{i m}(j, t), \rho_{c}(j, t), j=1, \ldots, N$, and $\phi(t)$ are solved. The widely differing time scales $[13,32,37]$ calls for appropriate care in the numerical solutions. We use a variable step fourth order Runge-Kutta scheme with an accuracy of $10^{-6}$ for all the four variables. The spatial derivative in $\rho_{m}$ is approximated by its central difference. The initial values of the dislocation densities are so chosen that they mimic the values in real samples. They are uniformly distributed with a Gaussian spread along the sample. However, for most calculations, we have used the steady state values for the variables as the long term evolution does not depend on the initial values. As for the boundary conditions, we note that the sample is strained at the grips. This means that there is a high density of immobile dislocations at the ends of the sample. We simulate this by employing two orders of magnitude higher values for $\rho_{i m}(j, t)$ at the end points $j=1$, and $N$ than the rest of the sample. Further, as bands cannot propagate into the grips, we use $\rho_{m}(j, t)=\rho_{c}(j, t)=0$ at $j=1$ and $N$.

For the original model $(D=0)$, it has been shown that the fixed point of the system of equations becomes unstable in a certain range of parameter values. In particular, as a function of the applied strain rate, the PLC state is reached through a Hopf bifurcation and is terminated by a reverse Hopf bifurcation ( with the other parameters kept in the instability domain). This feature is retained with the addition of the spatial degrees of freedom except that the number of complex conjugate roots are $2 \mathrm{~N}$, the negative ones are $\mathrm{N}$ and one zero exponent. We find that the instability domain in $\dot{\varepsilon}$ increases when the values of the other parameters $a, b_{0}, c, d, m$ are taken as in Ref. [37]. This is due to fact that the range of $\dot{\varepsilon}$ depends on the value of $D$ due to the global coupling in Eq. 6 ( The domain converges quickly as a function of $N$.) The boundary of $\dot{\varepsilon}$ is approximately in the range 10 to 1000 for $a=0.8, b_{0}=0.0005, c=0.08, d=0.00006, m=3.0, h=0$ with $D=0.5$, beyond which a uniform steady state exits. A set of four eigen values are shown in Fig. 1 The numerical results reported in the present work are for the above values. However, the results hold true for a wide range of values of other parameters in the instability domain including a range of values of $D$. Various system sizes are used depending upon the property studied, but are generally in the range $N=100$ to 3333. A sequence of values of $\mathrm{N}$ are used wherever convergence of the properties are investigated.

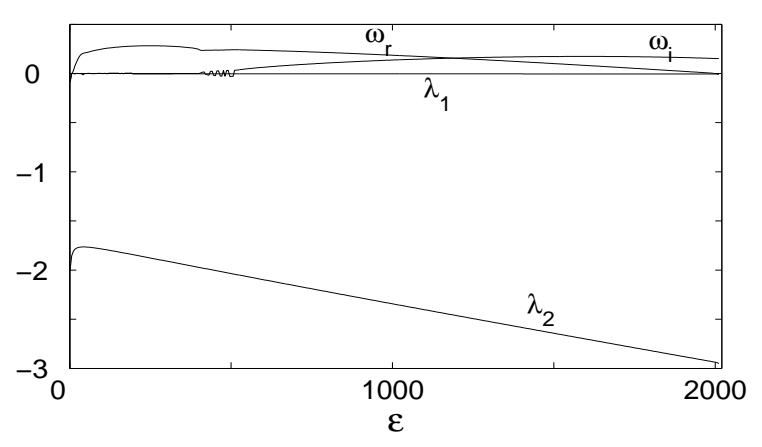

FIG. 1: Eigen value spectrum of the fixed point for the model. $\omega_{r}$ and $\omega_{i}$ refer to the real and imaginary parts of the eigen value.

\section{METHODOLOGY}

As our approach is fully dynamical and keeping in view the materials science community, we collect here a few definitions and provide some details of the methodology used in the analysis. Characterizing the dynamics of the model equations is carried out by studying the Lyapunov spectrum. The number of Lyapunov exponents $M$ for a given $N$ is $M=3 N+1$. We shall also use two other well know invariants namely the Kaplan-Yorke dimension $D_{K Y}=j+\frac{\sum_{i=1}^{j} \lambda_{i}}{\left|\lambda_{j+1}\right|}$, where $j$ is such that $\sum_{i=1}^{j} \lambda_{i}>0, \sum_{i=1}^{j+1} \lambda_{i}<0$ and the Kolmogorov entropy $H=\sum_{i=1}^{p} \lambda_{i}$ such that $\lambda_{p} \geq 0$ and $\lambda_{p+1}<0$. One important issue that is relevant to systems with many degrees of freedom is the existence of a limiting density for the Lyapunov spectrum as the system size is increased. This requires that we should ascertain if $\lambda_{j}$ versus $x=j / L^{d}$ converges to a welldefined asymptotic density function $\Lambda(x)$ with $x \in[0,1]$. ( See Ref. [27].) We address this issue by calculating the spectrum for various system sizes $N=100$ to 3333 which covers approximately two orders in $M$. In particular, such a study will be useful in comparing the results of our model with the GOY shell model for turbulence [28] in the power law regime of stress drops. Then, one expects that $j / D_{K Y}$ converges to well defined density function. Following Ref. [28], we use $j / D_{K Y}$ verses an appropriately scaled quantity $\lambda_{j} D_{K Y} / H$. This quantity is expected to converge to $f\left(\lambda_{j} D_{K Y} / H\right)$. ( We note here that the distribution function is proportional to the negative derivative of $f$.) The nature of the converged Lyapunov density function $f\left(\lambda_{j} D_{K Y} / H\right)$ as a function of the drive parameter $\dot{\varepsilon}$ can be used to quantify the changes the dynamics during the crossover.

As stated earlier, a proper description of the PLC effect requires a description of both the slow and fast time scales which in turn requires special techniques in nonlinear dynamics. These two time scales are transparent in the model equations where Eq. (1) represents a fast dynamics compared to the rest ( both Eq. (2) and (6) are slow while (3) falls in between). Such a system can be studied by eliminating the fast variable thereby allowing a reduction in the dimensionality of the system [38]. To illustrate this consider

$$
\mu \dot{x}=f(x, y, \mu)
$$




$$
\dot{y}=g(x, y, \mu)
$$

where $\mu$ is small parameter and $x \in \mathbb{R}^{p}$ and $y \in \mathbb{R}^{q}$. The main feature of such systems is that $x$ evolves much faster than $y$ unless $f(x, y, \mu)$ is small. In the vicinity of the slow manifold defined by $f(x, y, \mu)=0$, the dynamics is characterized by the evolution of the slow variable $y$. Thus, there is a reduction in the dimensionality of the system. On the other hand, if one is interested in the fast sub-system, using a scaled time $\tau=$ $t / \mu$, we get the corresponding fast variable $x$ defined by Eq. [7] where the slow variables $y$ act as parameters (obtained from Eq. 8). This subspace is clearly the complimentary subspace of the slow manifold. We shall use these two subspaces for the visualization of dislocation configurations in the high strain rate power law regime and obtain the band velocity at high strain rates respectively.

The analysis of the experimental stress-time series is carried out by estimating both correlation dimension $v$ and the Lyapunov spectrum. These methods involve embedding the scalar time series in a higher dimensional space using timedelay technique [18, 25]. Given a time series $\left\{\sigma_{j} \mid j=1, . ., M\right\}$, one first constructs vectors $\vec{\xi}_{i}=\left(\sigma_{i}, \sigma_{i-\tau}, \sigma_{i-2 \tau}, \ldots, \sigma_{M-(d-1) \tau}\right)$ in a $d$ dimensional space. The assumption here is that the actual dynamics can be unfolded by embedding the time series in a higher dimensional space in which the original attractor resides. (In addition, surrogate data analysis was also carried out in [19].) Then, a quantitative estimate of the self similarity of the attractor, namely the correlation dimension, $v$, can be obtained by calculating the integral [39] $C(r)=\frac{1}{N_{T}} \sum \Theta\left(r-\left|\vec{\xi}_{i}-\vec{\xi}_{j}\right|\right) \sim r^{v}$, where $N_{T}$ is the total number of points in the sum. Correlation dimension also provides a lower bound for the number degrees of freedom required for a dynamical description of the system which is given by the minimum integer larger than $v+1$ [40]. The geometrical interpretation of these degrees of freedom is that they correspond to the subspace to which the trajectories are confined. The dimension of this subspace can be obtained directly by using singular value decomposition (SVD) [41]. This method is often used for filtering noise component superposed on the time series. However, another use of SVD in the present context is that it is useful for the visualization of the strange attractor. (This method has been applied to the PLC time series earlier [16].) The method involves setting up the $m \times d$ trajectory matrix $\mathbf{T}$ defined by $\left(\vec{\xi}_{1}, \vec{\xi}_{2}, \ldots, \vec{\xi}_{m}\right)$ where $m=M-(d-1) \times \tau$. The eigen values of the matrix are obtained using the standard method of decomposition $\mathbf{T}=\mathbf{U W V}^{\mathbf{T}}$, where $\mathbf{U}$ is $m \times d$ orthogonal matrix, $\mathbf{V}$ is a $d \times d$ unitary matrix and $\mathbf{W}$ is the matrix of eigen values of the covariance matrix of $\mathbf{T}$ which are all nonnegative. The eigen values usually decrease rapidly saturating to a level below which the changes are minimal. Then the dimension of the attractor is taken to be that corresponding to number at which the eigen values saturate.
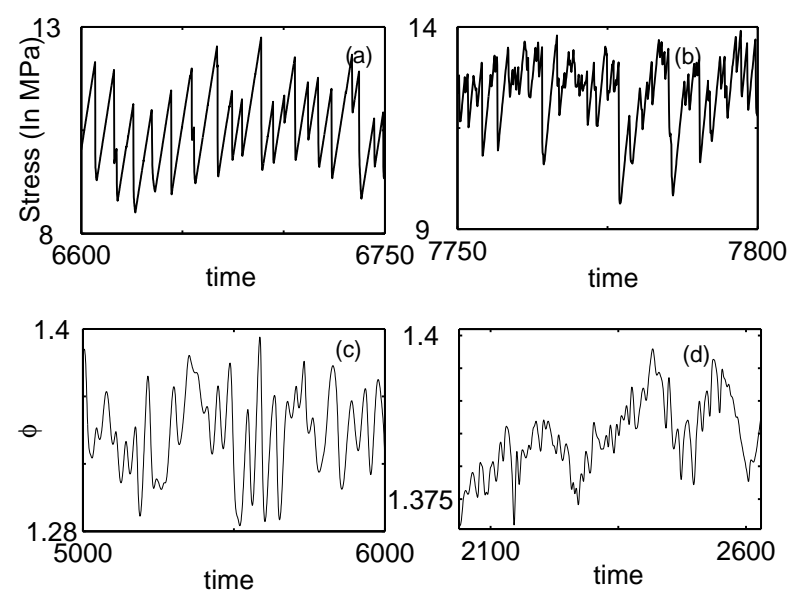

FIG. 2: (a) \& (b) Experimental stress-time series: (a) chaotic state at strain rates $\dot{\varepsilon}_{a}=1.7 \times 10^{-5} s^{-1}$ and (b) power law state at $\dot{\varepsilon}_{a}=$ $8.3 \times 10^{-5} s^{-1}$. (c) \& (d) Stress-time series from the model at (c) $\dot{\varepsilon}=120(\mathrm{~d}) \dot{\varepsilon}=280$.

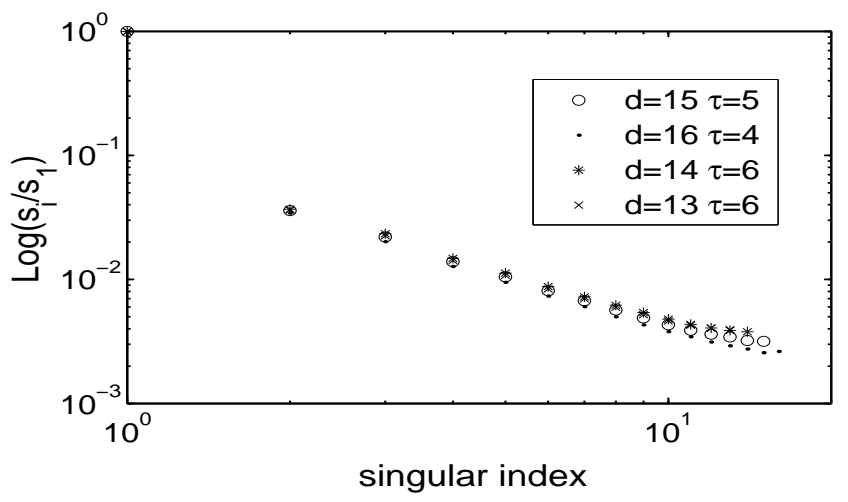

FIG. 3: Singular value spectrum of the experimental time series shown in Fig. 2,

\section{COMPARISON WITH EXPERIMENTS}

To make the motivation clear, we begin by briefly recalling the relevant experimental results on the crossover phenomenon and then compare them with those from the model. The simplest feature to compare is the nature of serrations in the respective regimes of strain rate. Plots of two experimental stress-strain curves from $\mathrm{CuAl}$ single crystals corresponding to the chaotic and power law regimes of applied strain rates are shown in Fig. 2 a,b. The stress-time series in the intermediate and high strain rate regimes from the model are shown in Fig. 22 ,d. The similarity of the experimental time series with that of the model in the respective regimes are clear.

The analysis of the stress-time series given in Fig. 2 has been reported in Ref. [19]. The correlation dimension was found to be $v=2.3$. Then, the number of degrees of freedom required for the description of the dynamics of the system given by the minimum integer larger than $v+1[40]$ is seen to be four, consistent with that used in the original model. As an 

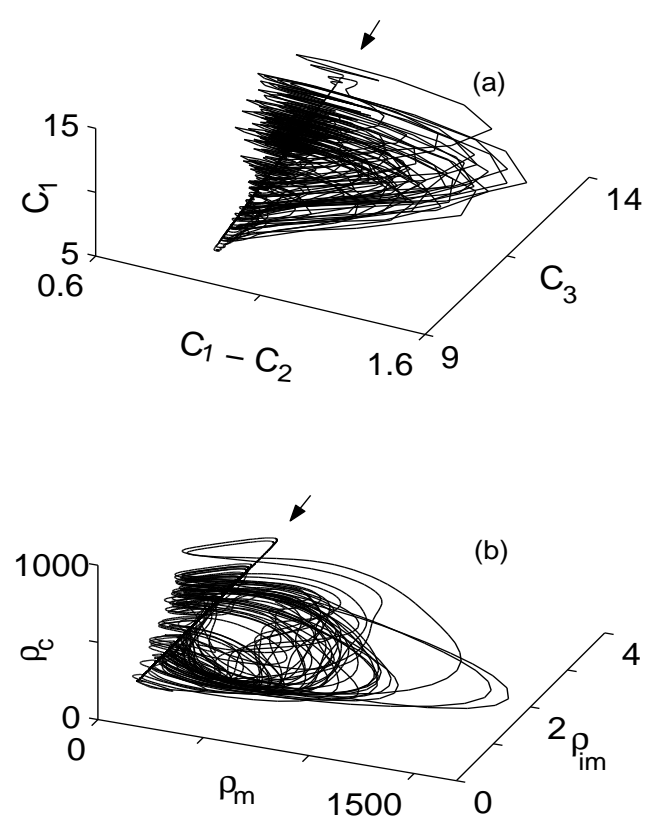

FIG. 4: (a) Reconstructed experimental attractor from the time series shown in Fig. 2a. (b)Attractor from the model for $N=100, j=50$.

independent check to obtain the number degrees of freedom, as also for the visualization of the experimental attractor, we have carried out the singular value decomposition of this time series. The normalized eigen values ( with respect the largest) is shown in Fig. 3. It is clear that the relative strength of the fourth eigen value drops more than two orders of magnitude compared to the first and changes very little beyond the fourth. Thus, we estimate the dimension of the experimental attractor to be four which is also consistent with that obtained from the correlation dimension. ( For time series from model systems, one usually finds a floor level below which the eigen values saturate. This is taken as the dimension of the actual attractor. However, in real situations, as in the present case, the eigen values do not saturate due to the presence of noise.) Then, for the visualization of the experimental attractor, we can use the dominant eigen values to reconstruct the nature of the attractor. Using the first three principal directions of the subspace $C_{i} ; i=1$ to 3 , we have reconstructed the experimental attractor in the space of specifically chosen directions $C_{1}-C_{2}, C_{3}$ and $C_{1}$ to permit comparison with the attractor obtained from the model. This is shown in Fig. 4 a for the experimental time series at $\dot{\varepsilon}_{a}=1.7 \times 10^{-5} s^{-1}$. This can be compared with the strange attractor obtained from the model in the space of $\rho_{m}, \rho_{i m}$ and $\rho_{c}$ (at an arbitrary spatial location, here $j=50$ and $N=100$ ) shown in Fig. 4 $\mathrm{b}$ for $\dot{\varepsilon}=120$ corresponding to the mid chaotic region (see below). Note the similarity with the experimental attractor particularly about the linear portion in the phase space (Fig. 4 a). This direction can be identified with the loading direction in Fig. 2]a. Note that the identification of the loading direction is consistent with the absence of growth of $\rho_{m}$.

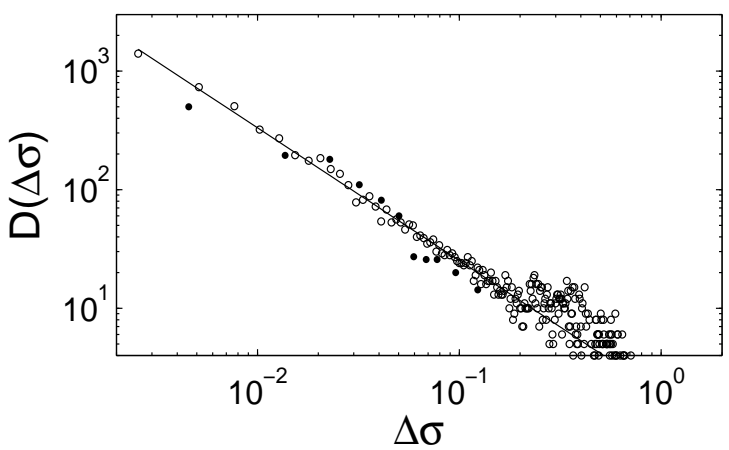

FIG. 5: Distributions of the stress drops from the model (०), from experiments $(\bullet)$ for $N=1000$ and $\dot{\varepsilon}=280$. Solid line is a guide to the eye.

In contrast to the experimental time series at low and medium strain rates, for the time series at the highest strain rate ( Fig. 2 [ b), we neither find a positive Lyapunov exponent and nor a converged value of the correlation dimension as was shown in Ref.[19]. However, the distribution of stress drops was shown to obey a power law [19].

Apart from comparing the statistics of stress drops from the model with that of the experimental time series, there is another more important issue, namely, does it generate power law statistics? If so, what mechanism is responsible for this? This is particularly important as the model is fully dynamical and noise free. It is clear that Fig $2 \mathrm{~d}$ is similar to Fig. 2 b, as there no inherent scale in the magnitudes of the stress drops in both cases and thus it is likely to also show a power law statistics. Indeed, the distribution of stress drop magnitudes, $D(\Delta \phi)$, shown in Fig. 5 -obtained from long runs for a large system size $(N=1000)$ shows a power law $D(\Delta \phi) \sim \Delta \phi^{-\alpha}$ over two orders of magnitude which increases with both length of stress series and system size. (Note that the value of $N$ here nearly three times larger than the results in Ref. [32], Fig. 3b and thus, the power law is well converged with respect to the system size. ) Surprisingly, experimental points $(\bullet)$ corresponding to $\dot{\varepsilon}_{a}=8.3 \times 10^{-5} s^{-1}$ also fall on the same curve with an exponent value $\alpha \approx 1$.1. (We have scaled the experimental points by a constant amount along both the axis to show that these points also fall on the same line.) The distribution of the duration's of the stress drops $D(\Delta t) \sim \Delta t^{-\beta}$ also shows a power law with an exponent value $\beta \approx 1.3$. The conditional average of $\Delta \phi$ denoted by $\langle\Delta \phi\rangle_{c}$ for a given value of $\Delta t$ behaves as $\langle\Delta \phi\rangle_{c} \sim \Delta t^{1 / x}$ with $x \approx 0.65$. The exponent values satisfy the scaling relation $\alpha=x(\beta-1)+1$ quite well. The exponent values remain unaltered in the region of strain rate $270<\dot{\varepsilon}<700$ we have investigated thus is independent of the value of the drive parameter. ( There are models of coupled map lattices that produce power laws where the exponent value depends on the drive parameter.) We now investigate the underlying causes leading to this power law. 


\section{DYNAMICS OF CROSSOVER}

\section{A. Lyapunov Spectrum}

Our next aim is to characterize the dynamics of this crossover. A natural tool for characterizing the crossover is to study the distribution of Lyapunov exponents as a function of the applied strain rate in the entire interval where the PLC effect is seen. Further, we also discuss the convergence properties of the Lyapunov spectrum as the system size is increased. In particular, this will be useful in examining the density of null ( nearly vanishing) exponents and also to compare our results with that of the GOY model of turbulence.

We have calculated the spectrum of Lyapunov exponents using the algorithm due to Benettin et al [42]. The exponent values reported here were obtained by averaging over 15000 time steps after stabilization with an accuracy of $10^{-6}$. We have used several system sizes ranging from $N=100,150,350,500,1000$, and 3333 which covers approximately two orders of magnitude in $M$, i.e., from 301 to 10000. A rough idea of the changes in the dynamics of the system can be obtained by studying the dependence of the largest Lyapunov exponent (LLE) as a function of the strain rate. The LLE converges fast as a function of the system size. For instance, we find that the LLE for $N=500$ looks much the same for a much smaller system size $\mathrm{N}=100$ given in Fig. 3a in Ref. [32]. The LLE becomes positive around $\dot{\varepsilon} \approx 35$ reaching a maximum at $\dot{\varepsilon}=120$, practically vanishing around 250 . ( Periodic states are observed in the interval $10<\dot{\varepsilon}<35$.) In the region $\dot{\varepsilon} \geq 250$, the dispersion in the value of the LLE is $\sim 5 \times 10^{-4}$ which is the same order as the mean. Thus, the LLE can be taken to vanish beyond $\dot{\varepsilon}=250$.

The study of the Lyapunov spectrum reveals that in the chaotic regime of strain rates, only a small proportion of the exponents are positive, an equal small number are close to zero value and a large proportion of the exponents are negative. The distribution of the Lyapunov exponents $D(\lambda)$, is shown for $N=1000$ in the inset of Fig. 6 for the strain rate, $\dot{\varepsilon}=120$. For this system size (with a total of exponents $M=3001$ ), the number of positive exponents is $\approx 6.2 \%$ of the total number of exponents and the null exponents are also $\approx$ $9 \%$. (For numerical purposes null exponents are taken to correspond to $|\lambda| \leq 5.2 \times 10^{-4}$.) These ratios remain the same for the larger system sizes used.

While $D(\lambda)$ reflects the distribution of Lyapunov exponents in various regions, for studying the convergence of the Lyapunov spectrum, plots of the density function $j / D_{K Y}=$ $f\left(\lambda_{j} D_{K Y} / H\right)$ are better suited. Further, these quantities have been used traditionally in the studies of extended dynamical systems [27]. A plot of $j / D_{K Y}$ verses $\lambda_{j} D_{K Y} / H$ for $\dot{\varepsilon}=120$ for $\mathrm{N}=500$ and 1000 is shown in Fig. 6 It is clear that while the density function has not yet converged for negative values of $\lambda_{j} D_{K Y} / H$, those for positive values are already converged. As we increase the strain rate beyond $\dot{\varepsilon}=180$, concomitant with the decrease in the value of the LLE, the number of null exponents increases. For instance, at $\dot{\varepsilon}=220$, for which the maximum Lyapunov exponent is small $\sim 0.0058$, the number of null exponents increases to $30 \% M$ (see inset of Fig. (7).

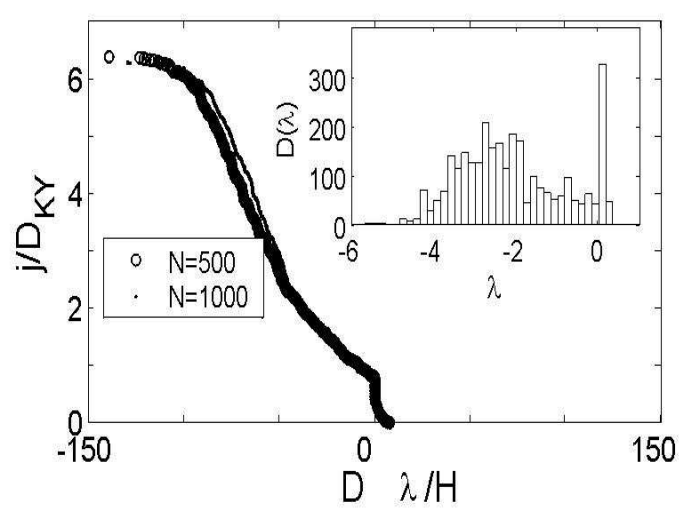

FIG. 6: A plot of $j / D_{K Y}$ verses $\lambda_{j} D_{K Y} / H$ for $\dot{\varepsilon}=120$. Inset shows a plot of $D(\lambda)$ as a function of $\lambda$ for $\mathrm{N}=1000$.

$D(\lambda)$ shows that the number of null exponents has increased. Concomitant with this trend, a plot of $j / D_{K Y}$ verses $\lambda_{j} D_{K Y} / H$ for $\mathrm{N}=500$ and 1000 (Fig. [7) shows that for $\dot{\varepsilon}=220$ is well converged for the entire range of values of the scaled Lyapunov exponent $\lambda_{j} D_{K Y} / H$. This signals a faster convergence of the density function $j / D_{K Y}=f\left(\lambda_{j} D_{K Y} / H\right)$ with the system size as we approach the scaling regime. Indeed, we find that plots for $\mathrm{N}=500$ and 1000 for strain rate $\dot{\varepsilon}=280$ cannot be distinguished over the entire range of values of $\lambda_{j} D_{K Y} / H$. Even though it would be adequate to use $N=1000$, for further analysis when dealing with Lyapunov spectrum in the scaling regime, we use a much bigger system size of $N=3333$, which for all practical purposes can be taken to be large $N$ limit. A plot of $j / D_{K Y}$ verses $\lambda_{j} D_{K Y} / H$ shown in Fig. 8 for $N=3333$ ( and also for 1000) shows that for $\dot{\varepsilon}=280$ is well converged for the entire range of values of $\lambda_{j} D_{K Y} / H$. Note also that nearly $40 \%$ the exponents are close to zero (see the inset).

As we approach the power-law regime of stress drops (extending from $\dot{\varepsilon}=250$ ), as the largest Lyapunov exponent approaches zero $\left(\sim 5.16 \times 10^{-4}\right.$ for $\left.\dot{\varepsilon}=280\right)$, exponents below a certain value cross each other as a function of time. However, the first few exponents remain distinct. Figure 9] shows the first two exponents that are well separated and another two which are close to each other in magnitude (for $\dot{\varepsilon}=280$ and $\mathrm{N}=3333$ ). Below $|\lambda|<5 \times 10^{-5}$, even though the exponents cross each other, the distribution of the exponents remains unchanged. The most significant feature of the spectrum in the region is that there is a dense set of null exponents. The peaked nature of the distribution of the null exponents $\left(|\lambda| \leq 5.2 \times 10^{-4}\right)$ for $\dot{\varepsilon}=280$ for $N=3333$ is shown in Fig. 10

The peaked nature of $D(|\lambda|)$ for null exponents suggests the possibility of a power law distribution for their magnitudes. We have plotted the distribution of the null exponents $\left(\left|\lambda_{i}\right| \leq\right.$ $5.2 \times 10^{-4}$ ) for $\dot{\varepsilon}=280$, for a system size of $N=3333, M=$ 10000 shown in Fig. 11 It is clear that both the positive and negative exponents show a power-law distribution $D(|\lambda|) \sim$ $|\lambda|^{-\gamma}$ with an exponent value $\gamma \sim 0.51$ and the scaling extends over an impressive three decades as shown in Fig.11 As null exponents correspond to marginal stable nature of the system, 


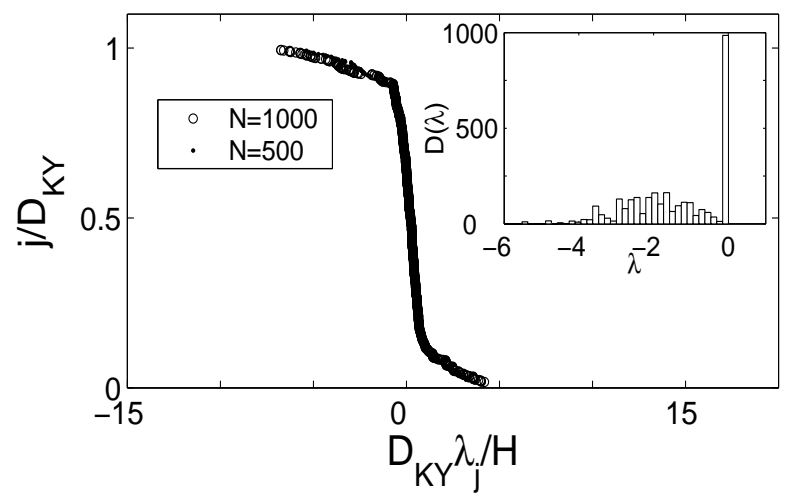

FIG. 7: A plot of $j / D_{K Y}$ verses $\lambda_{j} D_{K Y} / H$ for $\dot{\varepsilon}=220$. Inset shows a plot of $D(\lambda)$ as a function of $\lambda$ for $\mathrm{N}=1000$.

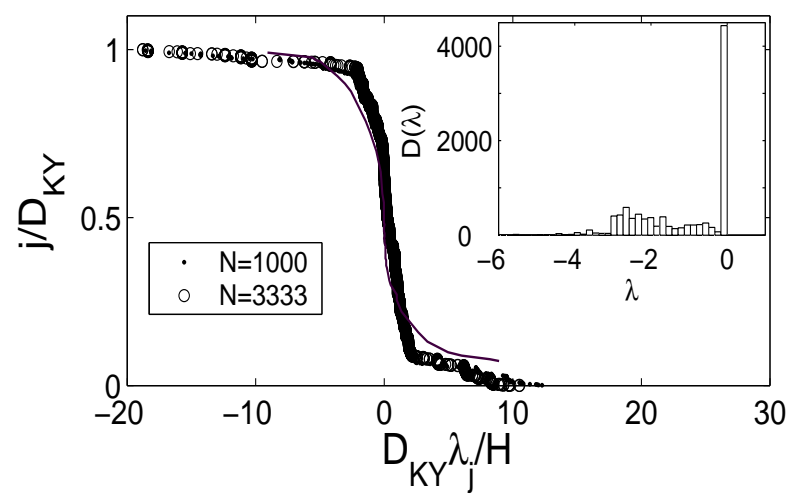

FIG. 8: A plot of $j / D_{K Y}$ verses $\lambda_{j} D_{K Y} / H$ for $\dot{\varepsilon}=280$. Inset shows a plot of $D(\lambda)$ for $\mathrm{N}=3333$. A schematic plot of the Lyapunov density function (continuous line) for the GOY model (after [28]).

their finite density, which itself obeys a power law, elucidates the underlying cause of power law distribution of stress drops at high strain rates.

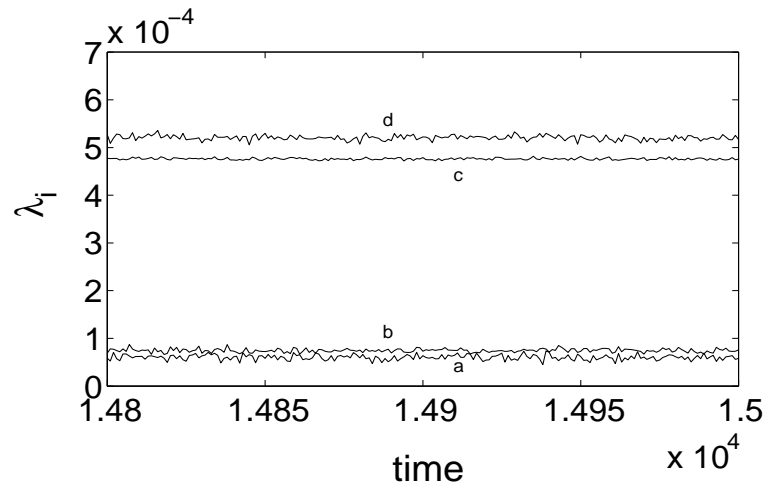

FIG. 9: The first two Lyapunov exponents that do not cross each other as a function of time for $\mathrm{N}=3333$ for $\dot{\varepsilon}=280$. Also shown are two more exponents that are close to each other.

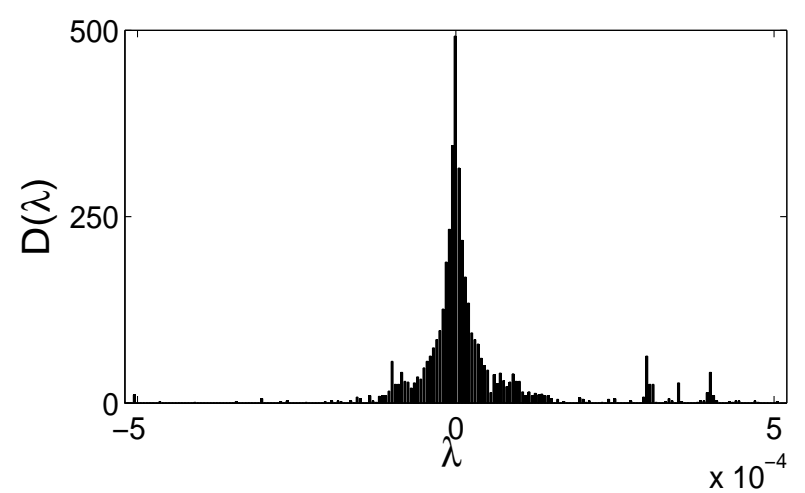

FIG. 10: The peaked nature of the distribution of null exponents lying in the range $\left[-5.2 \times 10^{-4}, 5.2 \times 10^{-4}\right]$ for $\dot{\varepsilon}=280, N=3333$.

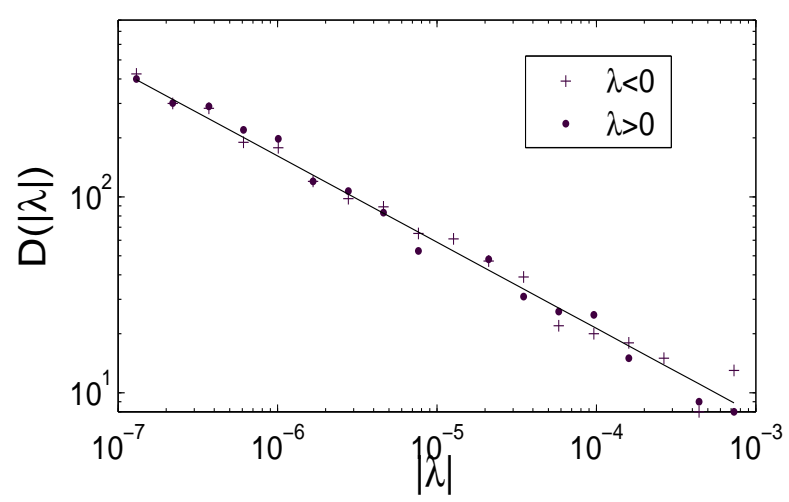

FIG. 11: Log-log plot of the distribution of the marginal exponents for $\dot{\varepsilon}=280, N=3333$. Solid line is a guide to the eye.

\section{B. Comparison with Shell Model for Turbulence}

As mentioned in the introduction, both in the PLC effect and in turbulence, power law statistics is seen at high drive rates in contrast to conventional SOC systems where the power law arises at low drives [23]. In addition, the dense set of null Lyapunov exponents in the scaling regime is similar to the finite density of null exponents obtained by Ruelle [43] for the discrete spectrum of the operator that linearizes the Navier-Stokes equations, if the fractal dimension of the energy dissipation set is $D_{F} \leq 2.5$. This property is preserved by the GOY shell model [28]. Here we attempt a comparison of the Lyapunov spectrum obtained from our model with that of the GOY model.

Shell models of turbulence [27] are designed to mimic the behavior of Navier-Stokes equations at high drives where the power law is seen. One standard model is the GOY model [27, 28]. For this model, Ohkitani and Yamada [28] gave a good numerical evidence that the density function exists as the viscosity parameter $\eta$ tends to zero. In our case, the role of the viscosity parameter is taken by the applied strain rate. In Section IV A, we have shown that there is a rapid increase in the density of null exponents and consequently, there is a rapid convergence of $j / D_{K Y}=f\left(\lambda_{j} D_{K Y} / H\right)$ as function of 
$\mathrm{N}$, starting from $\dot{\varepsilon}=220$. This suggests that one should expect convergence of the limiting $j / D_{K Y}=f\left(\lambda_{j} D_{K Y} / H\right)$ function as we approach the power law strain rate regime of stress drops. Thus, we should expect that the limiting distribution itself converges as a function of $\dot{\varepsilon}$ as we approach the scaling regime. Considering $N=1000$ approximates the limiting distribution ( see Fig. 7 for justification), we have verified that plots of $j / D_{K Y}=f\left(\lambda_{j} D_{K Y} / H\right)$ for three values of $\dot{\varepsilon}=250,260$ and 280 for reasonably large $\mathrm{N}=1000$ converge. This result is similar to the convergence of the density function in the GOY model as a function the viscosity parameter. The density function $j / D_{K Y}$ obtained from the model can be compared with that of the GOY model. Plot of $j / D_{K Y}=f\left(\lambda_{j} D_{K Y} / H\right)$ for a large system $\mathrm{N}=3333$ ( which can be taken to represent the limiting density as a function of system size ) for $\dot{\varepsilon}=280$ is shown in Fig. 8 along with a schematic plot for the GOY model shown by the continuous line. As can be seen, in both the cases, the distribution function which is proportional to $-d f(\lambda) / d \lambda$, shows a singularity near zero. The difference being that the singularity is more pronounced for our model. Ohkitani and Yamada also plot another quantity which represents the null exponents better, namely, the sum of Lyapunov exponents up to $j$ normalized by $H$ as function of $j$ scaled by $D_{K Y}$. The quantity $\sum_{1}^{j} \lambda_{j} / H$ is an increasing function of $j / D_{K Y}$ for positive $\lambda_{j}$ and goes to unity when $\sum_{i=1}^{j} \lambda_{i}=H$. In the region of null exponents, this quantity remains constant and then decreases with $j$ when $\lambda_{j}$ 's are negative. Thus, this quantity also reflects the density of null exponents. A schematic plot of $\sum_{1}^{j} \lambda_{j} / H$ as a function of $j / D_{K Y}$ ( continuous and dashed line) for the GOY model is shown in Fig. 12 The increase in $\sum_{1}^{j} \lambda_{j} / H$ for small $j / D_{K Y}$ shows that there is a finite density of positive exponents in the Lyapunov spectrum for the GOY model. Further, these authors find that there is a convergence with respect to the viscosity parameter for Lyapunov spectrum corresponding to the interior of the attractor (ie., $j / D_{K Y}<1$ ), while there is scatter for $j / D_{K Y}>1$ (the dashed line represents this portion). We have plotted $\sum_{1}^{j} \lambda_{j} / H$ as a function of $j / D_{K Y}$ for $\mathrm{N}=3333$ on the same plot for the sake of comparison. In our case, the increase to unit value is much slower ( compared to the GOY model) which clearly implies that there are very few positive exponents (of any significant magnitude) with most of them being vanishingly small. This feature is unlike the shell model where there is a finite density of positive exponents. In the GOY model, the largest exponent is proportional to $\eta^{-1 / 2}$ which is reflected in the steeper increase in $\sum_{1}^{j} \lambda_{j} / H$ for the GOY model.

Thus, we have shown that the power-law regime seen in our model at high drives as in hydrodynamics is not a superficial feature. The Lyapunov spectrum of the model is quite similar to the GOY model which however has a finite density of positive exponents. In addition, the distribution of the null Lyapunov exponents itself shows a power law in our case. Such a feature has not been verified for the GOY model. This feature is also quite distinct from the Lyapunov spectrum of models of SOC studied so far [44, 45, 46]. We comment on this later.

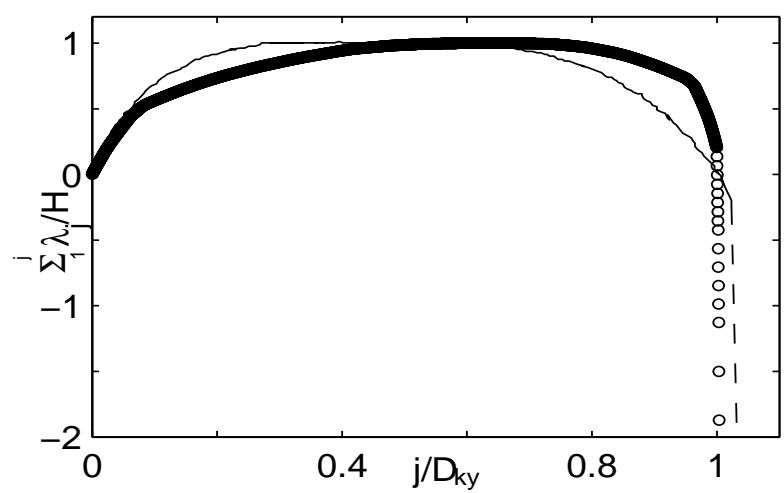

FIG. 12: A plot of $\sum_{1}^{j} \lambda_{j} / H$ as a function of $j / D_{K Y}$ for $\dot{\varepsilon}=280$ and $N=3333$. The corresponding schematic plot for the GOY model for $j / D_{K Y}<1$ (continuous line) and $j / D_{K Y} \geq 1$ (dashed curve) (after [28]).

\section{Slow Manifold Analysis}

The analysis provided in the previous section shows that as the strain rate is increased most exponents get concentrated in a narrow interval around the zero value. As zero Lyapunov exponents represent a marginal situation, we see that the region of strain rate beyond 240 (corresponding to the power law statistics of stress drops) can be identified with a marginally stable state. Thus, it would be interesting to realize a geometrical picture of dislocation configurations in the marginal state and examine how dislocations reach such state as a function of strain rate. We accomplish this through the slow manifold of the model [13, 37, 38].

Recently, the geometry of the slow manifold of the original model has been analyzed in detail [13, 37]. The analysis shows that the relaxational nature of the PLC effect arises from the atypical bent nature of the manifold. Here we recall some relevant results on the slow manifold of the original model $(D=0)$ and extend the ideas to the situation when the spatial degrees of freedom are switched on $(D \neq 0)$. Slow manifold expresses the fast variable in terms of the slow variables, conventionally done by setting the derivative of the fast variable to zero [13, 37]

$$
\dot{\rho}_{m}=g\left(\rho_{m}, \phi\right)=-b_{0} \rho_{m}^{2}+\rho_{m} \delta+\rho_{i m}=0 .
$$

where $\delta=\phi^{m}-\rho_{i m}-a$. The variable $\delta$ has been shown to have all the features of an effective stress and thus plays an important physical role [37], particularly in studying the pinning-unpinning of dislocations. We note that $\delta$ is a combination of two slow variables $\phi$ and $\rho_{\text {im }}$ both of which take small positive values. Hence, $\delta$ takes on small positive and negative values. Using Eq. 9 we get two solutions

$$
\rho_{m}=\left[\delta+\left(\delta^{2}+4 b_{0} \rho_{i m}\right)^{1 / 2}\right] / 2 b_{0},
$$

one for $\delta<0$ and another $\delta>0$. For regions of $\delta<0$, as $b_{0}$ is small $\sim 10^{-4}$, we get $\rho_{m} / \rho_{\text {im }} \approx-1 / \delta$ which takes on small values. This defines a part of the slow manifold, $S_{2}$ where $\rho_{m}$ is small. In this region, as the mobile density is small and 
immobile density is large ( relative to $\rho_{m}$ ), this region can be identified with pinned configuration of dislocations and hence we shall refer to the region $S_{2}$ as the 'pinned state of dislocations'. We note that larger negative values of $\delta$ correspond to strongly pinned configurations, as they refer to smaller ratio of $\rho_{m} / \rho_{i m}$. For positive values of $\delta$, another connected piece $S_{1}$ is defined by large values of $\rho_{m}$, given by $\rho_{m} \approx \delta / b_{0}$, which we refer to as the 'unpinned state of dislocations' as $\rho_{\text {im }}$ is also small. These two pieces $S_{2}$ and $S_{1}$ are separated by $\delta=0$, which we refer to as the fold line $[13,37]$ (see below). A plot of the slow manifold in the $\delta-\rho_{m}$ plane is shown in Fig. 13]. For the sake of illustration, we have plotted a monoperiodic trajectory describing the changes in the densities during a loading-unloading cycle. The inset shows $\rho_{m}(t)$ and $\phi(t)$. For completeness, the corresponding plot of the slow manifold in the $\left(\rho_{m}, \rho_{i m}, \phi\right)$ space is shown in Fig. 13 , along with the trajectory and the symbols. In this space, one can see that $\delta=\phi^{m}-\rho_{i m}-a=0$ is a line that separates the pieces $S_{2}$ and $S_{1}$ of the slow manifold, and hence the name fold line. The cyclic changes in the variables is well captured by the nature of trajectory shown in Fig. $13 \mathrm{~b}$. The trajectory enters $S_{2}$ at $A$ and moves into $S_{2}$, the value of $\delta$ ( in Fig. 13 ) decreases from zero to a maximum negative value as the trajectory reaches $B$. Then $\delta$ increases as the trajectory returns to $A^{\prime}$ before leaving $S_{2}$. The corresponding segment is $A B A^{\prime}$ in Fig. 13b, which is identified with the flat region of $\rho_{m}(t)$ in the inset of Fig. 13. As the trajectory crosses $\delta=0, \partial g / \partial \rho_{m}$ becomes positive and it accelerates into the shaded region (Fig. 13 ) rapidly till it reaches $\rho_{m}=\delta / 2 b_{0}$. Thereafter it settles down quickly on $S_{1}$ decreasing rapidly till it reenters $S_{2}$ again at $A$. The burst in $\rho_{m}$ (inset in Fig. 13 ) corresponds to the segment $A^{\prime} D A$ in Fig. 13 and $b$. The nature of trajectories for higher strain rate remains essentially the same, but is chaotic. The nature of the trajectories in the power law regime of strain rates which will be discussed later.

Having identified the regions of the slow manifold with the pinned and unpinned states of dislocations, we now consider the variation of stress when dislocations are pinned and are unpinned. Consider the stress changes as the state of the system goes though a burst of plastic activity. For $D=0$, Eq. 6 reduces to

$$
\dot{\phi}=d\left[\dot{\varepsilon}-\dot{\varepsilon}_{p}\right]
$$

where $\dot{\varepsilon}_{p}=\phi^{m} \rho_{m}$ defines the plastic strain rate. Since $\rho_{m}$ is small and nearly constant on $S_{2}$, stress increases monotonically. However, during the burst in $\rho_{m}$ ( $A^{\prime} D A$ in the inset), $\dot{\varepsilon}_{p}(t)$ exceeds $\dot{\varepsilon}$ leading to an yield drop. Since $\rho_{m}$ grows outside $S_{2}, \delta=0$ line separates the pinned state from the unpinned state. Thus, $\delta=0$ physically corresponds to the value of the effective stress at which dislocations are unpinned.

When the spatial degrees of freedom are included, there is no additional complication as the slow manifold is defined at each point. In this case, a convenient set of variables for visualization of dislocations is $\left(\rho_{m}(x), \delta(x), x\right)$. Here, our aim is to investigate the nature of typical spatial configurations in the chaotic and the power law regimes of stress drops and study the changes as we increase the strain rate. For simplicity, we shall use $h=0$ for which we have $\phi_{\text {eff }}=\phi$. ( It is straightfor-
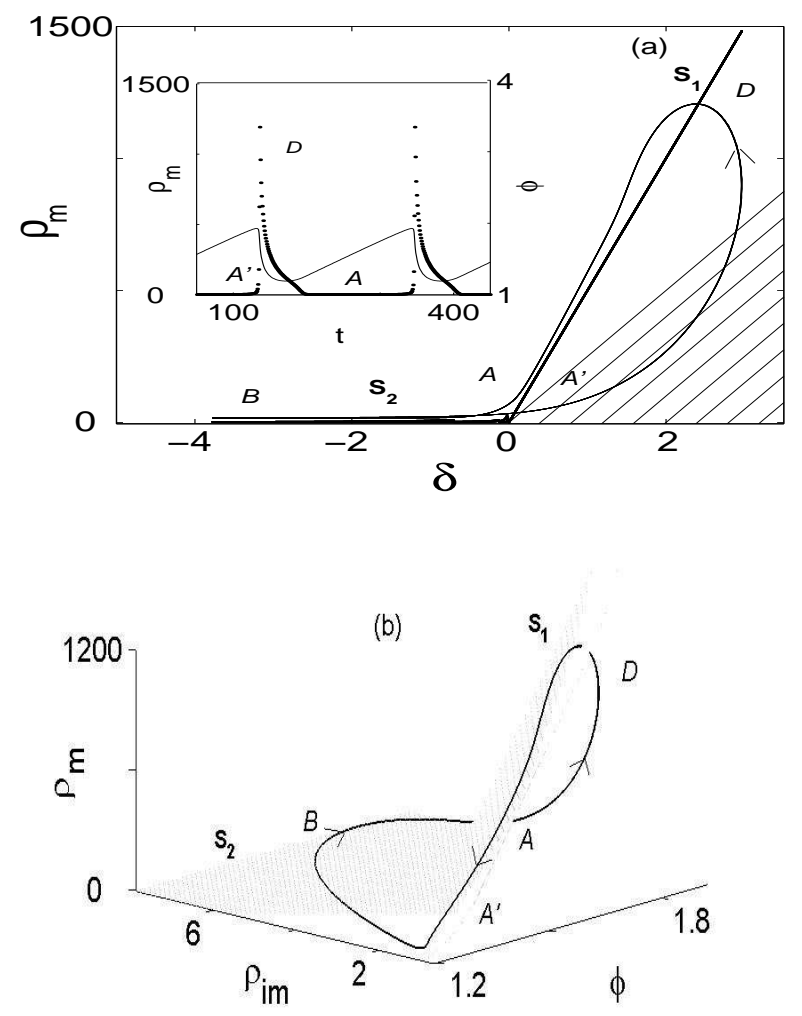

FIG. 13: (a)Bent slow manifold $S_{1}$ and $S_{2}$ (thick lines) with a simple trajectory for $\dot{\varepsilon}=200$ and $m=3$. Inset: $\rho_{m}$ (dotted curve) and $\phi$ (solid line).(b)The same trajectory in the $\left(\phi, \rho_{i m}, \rho_{m}\right)$ space.

ward to extend the arguments to the case when $h \neq 0$.) Then, the plastic strain rate $\dot{\varepsilon}_{p}(t)$ is given by

$$
\dot{\varepsilon}_{p}(t)=\phi^{m}(t) \frac{1}{l} \int_{0}^{l} \rho_{m}(x, t) d x=\phi^{m}(t) \bar{\rho}_{m}(t),
$$

where $\bar{\rho}_{m}(t)$ is the mean mobile density $\left(=\sum_{j} \rho_{m}(j, t) / N\right.$ in the discretized form). With the inclusion of spatial degrees of freedom, the yield drop is controlled by the spatial average $\bar{\rho}_{m}(t)$ rather than by individual values of $\rho_{m}(j)$. Further, we note that the configuration of dislocations change during one loading-unloading cycle. However, one should expect that configurations will be representative for a given strain rate. Further, we know that the drastic changes occurs during an yield drop when $\bar{\rho}_{m}(t)$ grows rapidly. Thus, we focus our attention on the spatial configurations on the slow manifold at the onset and at the end of typical yield drops.

First consider the configuration seen just before and after the yield drop when the strain rate is in the chaotic regime. In this regime, the stress drop magnitudes are large which implies that the change in mobile density is large. Figures 14 a, b for a typical value of $\dot{\varepsilon}=120$. It is clear that both at the onset and at the end of a typical large yield drop, the $\delta(j)$ values which reflect the state of system ( pinned or unpinned state), is negative and correspondingly the mobile density $\rho_{m}(j)$ 's are small, i.e., most dislocations are in a strongly pinned state. 

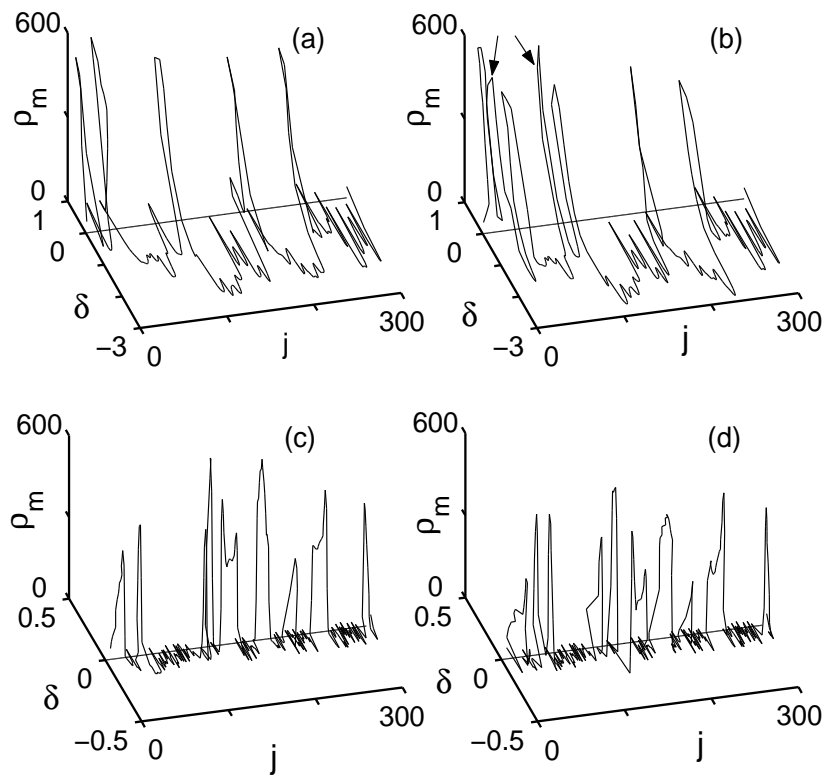

FIG. 14: Dislocation configurations on the slow manifold at the inset and at the end of an yield drop: (a) and (b) for $\dot{\varepsilon}=120$ (chaotic regime), and (c) and (d) for $\dot{\varepsilon}=280$ (scaling regime).

( Recall that $\delta$ signifies how close the spatial elements are close to unpinning threshold.) The arrows show the increase in $\rho_{m}(j)$ at the end of the yield drop. We have checked that this is a general feature for all yield drops in the chaotic regime of strain rates. Now consider dislocation configuration in the scaling regime at high strain rates, say, $\dot{\varepsilon}=280$, at the onset and at the end of an yield drop shown in Fig. 14 c,d respectively. In contrast to the chaotic regime, in the scaling regime, most dislocations are clearly seen to be at the threshold of unpinning with $\delta(j) \approx 0$, both at the onset and end of the yield drop. This also implies that they remain close to this threshold all through the process of an stress drop. We have verified that the edge-of-unpinning picture is valid in the entire power law regime of stress drops for a range of $N$ values. Further, as a function strain rate, we find that the number of spatial elements reaching the threshold of unpinning $\delta=0$ during an yield drop increases as we approach the scaling regime.

\section{TYPES OF BANDS}

The fact that the spatially extended Ananthakrishna's model is able to successfully reproduce the crossover dynamics from chaos to the power-law regime of stress drops ( and other generic features demonstrated earlier) might suggest that the characteristic features of the PLC bands may also emerge out of the model. Most models of dislocations bands use diffusive coupling although the physical mechanism of the term is different in different situations [1]. An important feature of the spatial coupling in the model is that it accounts for spreading of dislocations into regions of low back stress once dislocations are unpinned ( the factor $\rho_{i m}^{-1}$ ). The term also determines the length scale over which dislocations spread into the neigh-

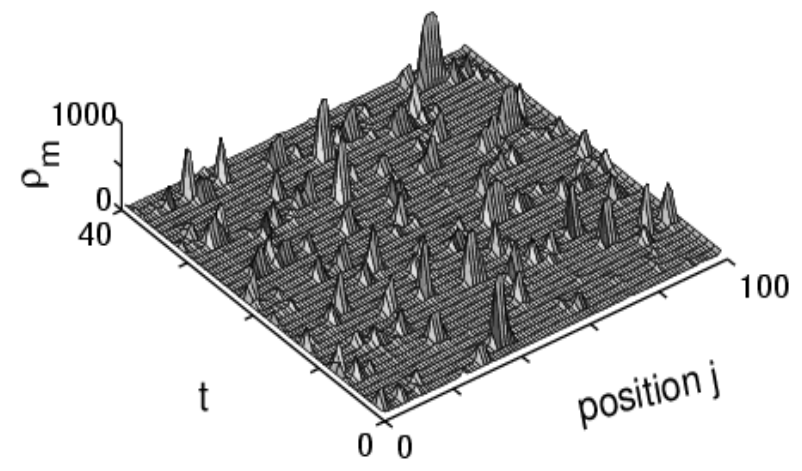

FIG. 15: Spatially uncorrelated bands at $\dot{\varepsilon}=40$.

boring elements. Thus, while dislocation pinning and unpinning gives a heterogeneity in space (in principle), regions of low $\rho_{i m}$ are favored for dislocation multiplication and spreading into neighboring regions. Further, this type of spatial term couples length scale and time scales in a dynamical way as $\rho_{i m}$ itself evolves in time and hence the associated time scale. Indeed, multiplication of dislocation depends on stress, (i.e., $\phi_{e f f}^{m}$, and hence this rate itself is changing dynamically leading to changes in the time scale of internal relaxation as a function of $\dot{\varepsilon}$. We expect this to lead to changes in spatial correlation as strain rate is increased.

Below we report both numerical and analytical studies on the spatiotemporal patterns emerging from the model as a function of the strain rate, $\dot{\varepsilon}$. We begin with the numerical results [47].

For $\dot{\varepsilon}<10$ and $\dot{\varepsilon}>2000$, we get homogeneous steady state solutions for all the dislocation densities, $\rho_{m}, \rho_{i m}$ and $\rho_{c}$. In these ranges of strain rates, $\phi$ takes the fixed point values asymptotically. In the region where interesting dynamics of chaotic and power law states are observed, the nature of the dislocation bands can be broadly classified into three different types occurring at low, intermediate and high strain rates described below.

For strain rates, $30 \leq \dot{\varepsilon}<70$, we get uncorrelated static dislocation bands. The features of these bands are illustrated for a typical value, say for $\dot{\varepsilon}=40$. A plot of $\rho_{m}(j, t)$ is given in Fig. 15 Dislocation bands of finite width nucleate randomly in space and they remain static till another band is nucleated at another spatially uncorrelated site. The associated stresstime curves which are nearly regular have large characteristic stress drops. The distribution of these stress drops is found to be peaked as in experiments at low strain rates [20].

At slightly higher values of strain rates, $70 \leq \dot{\varepsilon}<180$ we find that new bands nucleate ahead of the earlier ones, giving a visual impression of hopping bands. This can be clearly seen from Fig. 16 where a plot of $\rho_{m}(j, t)$ is given for $\dot{\varepsilon}=$ 130. However, this hopping motion does not continue till the other boundary. They stop midway and another set of hopping bands reappear in the neighborhood. Often nucleation occurs 


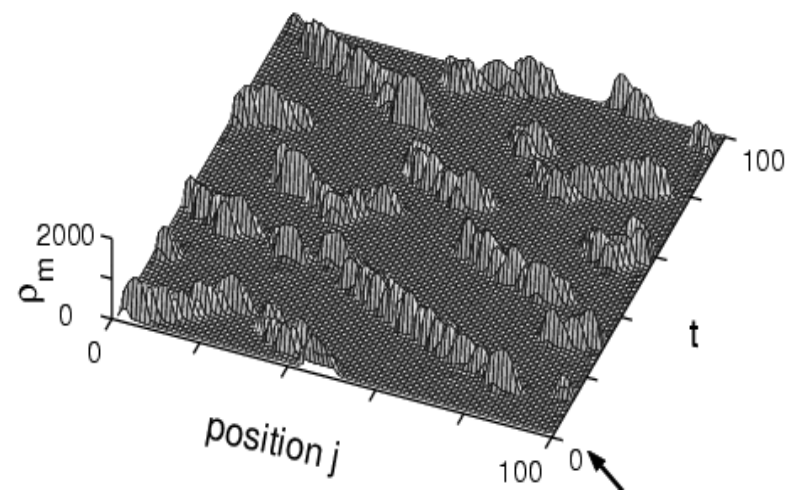

FIG. 16: Hopping type bands at $\dot{\varepsilon}=130$ (arrow shows one such band).

at more than one location. Stress-time plots in this regime have a form similar to Fig. 2k with the average amplitude of the stress drops being smaller than the localized non-hopping bands at low strain rates as seen in experiments. These stress drops also have a nearly symmetric peaked distribution as in the previous case but slightly skewed to the right similar to those observed in experiments [20].

As the strain rate is increased further, the extent of propagation increases, concomitantly, the magnitudes of the stress drops decrease. We see continuously propagating bands even at $\dot{\varepsilon}=240$ as can be seen from Fig. 17 One can see dislocation bands nucleating from one end of the sample $(j=0, t=$ 25,50 and 75) and propagating continuously to the other end. Often, we see a band nucleating at a point, branching out and propagating only partially towards both the ends. Unlike the present case which exhibits rather uniform values of $\rho_{m}$, we usually find irregularities as the band reaches the edges. The stress strain curves in this region of strain rates, exhibit scale free feature in the amplitude of the stress drops (Fig. 2]d) with a large number of small drops. As can be seen from Fig. 2] d, the mean stress level of these small amplitude stress drops increases until a large yield drop is seen. This large stress drop corresponds to bands having reached the end of the specimen.

It is possible to calculate the velocity of the propagating bands in the high strain rate limit. We first note that our equations constitute a coupled set of integro-partial differential equations, and hence cannot be dealt with in their present form. To reduce these equations to a form that is suitable for further analysis, we recall a few pertinent points about the changes in the structure of the slow manifold as a function of the applied strain rate. We note that the original model exhibits an incomplete approach to homoclinicity [37], i.e., the number of mixed mode oscillations of the type $L^{s}$, where $s$ refers to the small period oscillations and $L$ refers to a large relaxation oscillation, are limited. Typically, about 12 small period nearly harmonic oscillations are known to occur for a single large one at high values of the strain rate. The reason at-

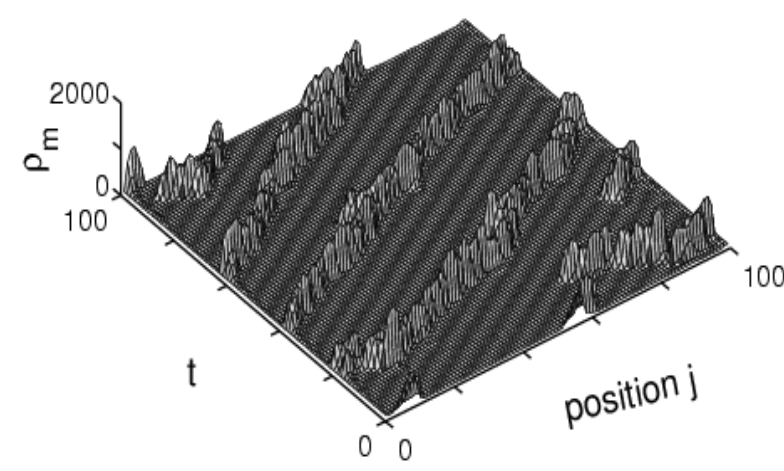

FIG. 17: Fully propagating bands at $\dot{\varepsilon}=240$

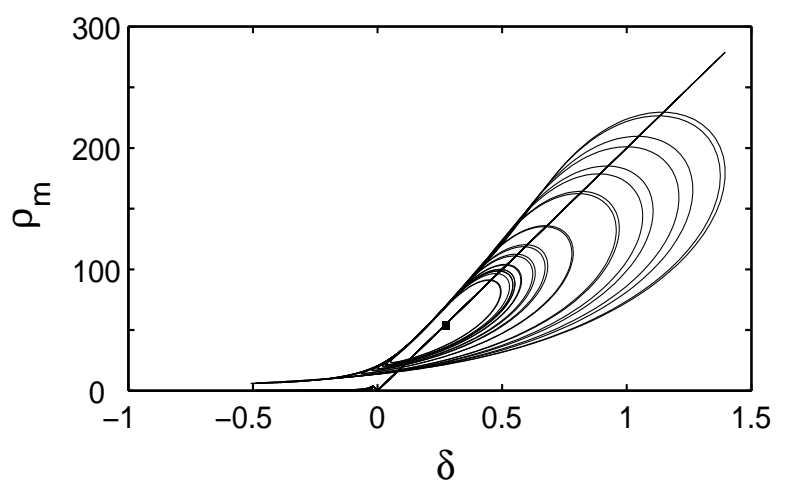

FIG. 18: Slow manifold showing a trajectory for the space independent model near the reverse Hopf bifurcation point, at $\dot{\varepsilon}=90, m=2$. - fixed point of Eqn 1-3 and 6.

tributed to this is the finite rate of softening of the eigen value of the fixed point due to presence of the reverse Hopf bifurcation is reached [37]. In the presence of the spatial coupling we find that the softening is further enhanced as is clear from the fact that the upper Hopf bifurcation is pushed to much larger values of strain rate ( $\dot{\varepsilon}_{c_{2}}=2000$, see Fig. 1). This enhanced softening rate implies that the number of small period oscillations is also increased in this domain of strain rates. Even so, the geometry of the slow manifold is not altered from that of the space independent model. In particular, the position of the unstable saddle focus remains located on the $S_{1}$ part of the manifold (Ref.[13, 37]). In addition, the feature of the fixed point approaching the fold line as a function of the strain rate is retained. Under these conditions, for high strain rates nearly sinusoidal oscillations are executed around the fixed point with the orbits touching $S_{2}$ only after executing several such turns. A plot of this is shown in Fig. 18 To understand the dynamics at high strain rates, we recall that our analysis in Ref. [37] shows that the orbit is re-injected along the stable manifold close to the unstable saddle focus ( as shown in Fig.11 of Ref.[37]). The orbit then spirals out along the unstable manifold of the fixed point. Once the orbit is sufficiently 
away from the fixed point when the influence of the fixed point is lost, it is re-injected close to the fixed point via $S_{1}$. The dynamics then repeats. Note that at high applied strain rate, the system is close to reverse Hopf bifurcation point and hence the fixed point is close to the applied strain rate value. Thus as the orbit executes one turn, there is one small yield drop. However, the orbit executes several turns around the fixed point, each turn leading larger loop sizes, i.e., larger values of $\rho_{m}$ and consequently to successively larger stress levels than the earlier one before briefly visiting $S_{2}$.

Under these conditions the dynamics is entirely controlled by the spiralling motion around the fixed point. Thus, the entire dynamics is essentially described by the fast variable; the other two variables $\rho_{i m}$ and $\phi$ can be taken to be parameters. Such a situation is described by the transient dynamics dictated entirely by equation of the fast variable (the so called layer problem [38]) and thus, we are justified in using only the evolution equation of the fast variable in terms of the slow manifold parameter $\delta=\phi^{m}-\rho_{i m}-a$. Since the trajectory rarely visits the $S_{2}$ part of the slow manifold, we restrict the calculations to $\delta>0$. The physical picture of a propagating solution is that as the orbit at a site makes one turn around the fixed point, i.e., $\delta$ small but positive, around the value of the applied strain rate, the front advances by a certain distance along the specimen like the motion of a screw.

The rate equation for the mobile dislocation density $\rho_{m}$ in terms of $\delta$ is

$$
\frac{\partial \rho_{m}}{\partial t}=-b_{o} \rho_{m}^{2}+\delta \rho_{m}+\rho_{i m}+D^{\prime} \frac{\partial^{2} \rho_{m}}{\partial x^{2}}
$$

where $D^{\prime}=D \phi^{m} / \rho_{\text {im }}$. Since, the slow variables, $\rho_{\text {im }}$ and $\phi$ are treated as parameters, this has the form of Fisher-Kolmogorov equation for propagating fronts, which has been well studied. This equation can be reduced to the standard form

$$
\frac{\partial Z}{\partial t^{\prime}}=Z(1-Z)+D^{\prime} \frac{\partial^{2} Z}{\partial x^{2}},
$$

(This is done by first transforming $\rho_{m}=X-\rho_{\text {im }} / \delta$, dropping the term $2 b_{0} \rho_{i m} / \delta$ compared to $\delta$ in the linear term in $X$, and then using $Z=X \delta / b_{0}$ and $t^{\prime}=t \delta$.) It is clear that $Z=0$ is unstable and $Z=1$ is stable. Using the form for propagating front $Z=Z_{o} e^{\omega t^{\prime}-k x^{\prime}}$, the marginal velocity is calculated using $v^{*}=\operatorname{Re} \omega\left(k^{*}\right) / \operatorname{Re}^{*}=d \omega /\left.d k\right|_{k=k^{*}}$ and $\mathfrak{I} d \omega /\left.d k\right|_{k=k^{*}}=0$, gives the velocity of the bands $v^{*}=2$ [48, 49]. In terms of the variables in Eqn. 13, the marginal velocity is

$$
v^{*}=2 \sqrt{D \delta} .
$$

In order to relate this to the applied strain rate, we note that for a fixed value of the strain rate (where propagating bands are seen), the average level of stress drop is essentially constant. Thus, from Eqn. 6 we see that in this regime of high strain rates, the applied strain rate $\dot{\varepsilon}$ is essentially balanced by the plastic strain rate $(1 / l) \int_{0}^{l} \phi^{m} \rho_{m}(x, t) \equiv \dot{\varepsilon}_{p}$. Then, using $\phi^{m}=$ $\dot{\varepsilon} / \bar{\rho}_{m}$, and using $\delta=\phi^{m}-a-\rho_{\text {im }}$, we get

$$
v=2 \sqrt{\frac{D \dot{\varepsilon}}{\bar{\rho}_{m} \rho_{i m}}\left(\frac{\dot{\varepsilon}}{\bar{\rho}_{m}}-a-\rho_{i m}\right)} .
$$

It is important to note that at high applied strain rate $\bar{\rho}_{m} \sim \bar{\rho}_{m}^{*}$ , the fixed point value. Thus, for all practical purposes, we can assume $\bar{\rho}_{m}$ as a constant. From the above equation, we see that the velocity of the propagating bands is proportional to the applied strain rate. This result is similar to the result obtained recently by Hähner et al. [50]. Further, $v \propto \bar{\rho}_{m}^{-1}$ which also appears to be consistent with an old experimental result. (See Fig. 7 of Ref. [51] which appears to fit $v \bar{\rho}_{m}=$ constant.) This result needs further experimental support.

As the form of our equation has been reduced to the standard form, all other results carry through, including nonlinear analysis. We have numerically calculated the velocity of the continuously propagating bands at high strain rates from the model which confirms the linear dependence of the band velocity on applied strain rate. In the region of strain rates $\dot{\varepsilon}=220$ to 280 (corresponding to unscaled strain rate values $\left.10^{-5}-1.5 \times 10^{-5} s^{-1}\right)$, we find that the unscaled values of the band velocity increases from 100 to $130 \mu \mathrm{m} / \mathrm{s}$. These values are consistent with the experimental values reported by Hähner et. al. [50].

We note here that the types of the bands seen in our model are correlated with the two distinct dynamical regimes investigated. The hopping type bands belong to the chaotic regime, a result consistent with the recent studies on $\mathrm{Cu}-\mathrm{Al}$ polycrystals [20]. On the other hand, the propagating bands are seen in the power law regime of stress drops [32], again consistent with these studies [19, 20]. Curiously the uncorrelated bands predicted by the model also belong to the chaotic regime. We shall now explain these results based on the dynamics of the model. We first note that each spatial element is described the three dislocation densities ( Eq. (1-3)). Consider one of these elements being close to unpinning threshold, ie., $\delta=0$. It has been shown earlier that $\rho_{i m}$ is out of phase with $\rho_{m}$ [13, 37]. This feature is retained with the spatial coupling as well. When the orbit is about to leave $S_{2}$, ie., when $\rho_{m}(j)$ is at the verge of a sharp increase, $\rho_{i m}$ is largest. However, the extent of the spatial coupling is determined by $\rho_{i m}^{-1}$. But the magnitude of $\rho_{i m}$ itself decreases with the applied strain rate, being large at low strain rates [13, 37]. Thus, the spatial width of this is small at low $\dot{\varepsilon}$ and large at high $\dot{\varepsilon}$. Next we note that the growth and decay of $\rho_{m}(j)$ with $j$ occurs over a short time scale which is typically of the order of the correlation time, $\tau_{c}$, of $\phi(t)$. Beyond this time, the memory of its initial state is lost. Consider an initial state when a band is formed at some location. Before the memory of this initial state decays, if a new band is not created, we get an uncorrelated band. On the other hand, if a new band is created before the memory of the initial state decays, there are two possibilities. If another band is created just before the correlation decays substantially by that time, we get a hopping type band. If however, even before the burst of $\rho_{m}(j)$ decreases beyond its peak value, new sources of creation of $\rho_{m}$ occur, then we end up seeing a propagating band. An analysis of the correlation time shows that it increases with the applied strain rate. Concomitantly, $\rho_{\text {im }}$ decreases with $\dot{\varepsilon}$ which implies that the spatial correlation increases. (Indeed, the value $\rho_{i m}$ is quite small for large $\dot{\varepsilon}$ as we reach the power law regime of stress drops.) Under these conditions, only partial plastic relaxation 
is possible in this regime. This discussion clarifies the dynamic interplay of time scales and length scales. Moreover, as the spatial coupling term allows the spreading of dislocations only into regions of low $\rho_{i m}$ or low back stress, the propensity for continuous propagation of the band is enhanced when $\rho_{i m}$ is small. In addition, we find that higher values of $\rho_{i m}$ at the wake of the band which favors propagation into regions of smaller immobile density thus determining the direction of propagation also.

\section{SUMMARY AND CONCLUSIONS}

We first summarize and make appropriate comments wherever necessary. Detailed numerical and analytical studies on the extended Ananthakrishna's model shows that it reproduces all the important features of the PLC effect including the crossover in the dynamics from a chaotic to a power law regime observed in experiments. It also provides insight into the dynamical causes leading to this crossover. A systematic study of the system size effects of the Lyapunov spectrum carried out elucidates the underlying mechanism controlling the crossover. The study demonstrates that the limiting Lyapunov distribution evolves from a set of positive and negative exponents with a few null exponents to a dense set of null exponents as we approach the scaling regime of stress drops. This study is complemented through an analysis of the slow manifold. This method is particularly useful in giving a geometrical picture of the spatial configurations, both in the chaotic and power law regime of stress drops. The study shows that the configuration of dislocations is largely in the pinned state in low and medium strain rates (chaotic domain) are pushed to the threshold of unpinning as we increase strain rate ( power law stress drop regime). The study also establishes that the present model has considerable similarities with the GOY model of turbulence [28]. The model also reproduces the major spatial features of the PLC effect. The randomly nucleated band, the hopping and propagating types are found as the strain rate is increased. It also predicts a linear dependence of the velocity of the band and inverse dependence on the mobile density at high strain rates.

Several observations may be in order on the dynamics of the crossover. We first note that the crossover itself is smooth as the changes in the Lyapunov spectrum are gradual, though it occurs in a narrow interval of strain rates ( from 220-250). Second, the power law here is of purely dynamical origin (in the sense elaborated below below). We have shown that this is a direct result of the existence of a reverse Hopf bifurcation at high strain rates. In this regime due to softening of the eigen values ( as a function of the applied strain rate), the orbits are mostly restricted to the region around the saddle node fixed point located on the $S_{1}$ part of the manifold. This offers a dynamical reason for the smallness of the yield drops in this region [13, 37]. Note also that there is a dynamic feed back between the stress determined by Eq. 6 and the production of dislocations in Eq. 11 which provides an explanation for the slowing down of the plastic relaxation. ( The partial plastic relaxation has been cited as the reason for the power law
[19, 20].) This sets up a competition between the time scale of internal relaxation and the time scale determined by the applied strain rate ( essentially Deborah number). We note that while the time scale for internal relaxation is increasing, that due to the applied strain rate is decreasing. Third, our analysis shows that the power law regime of stress drops occurring at high strain rates belongs to a different universality class compared to SOC systems, as it is characterized by a dense set of null exponents. This must be contrasted with the lack of any characteristic feature of the nature of the Lyapunov spectrum in the few models of SOC studied so far [44, 45, 46]. For instance, no zero and positive exponents, zero exponent in the large $N$ limit etc., have been reported [44, 45, 46]. (Often, the nature of largest Lyapunov exponent is inferred based on the similarity of other dynamical invariants [44].) The dense set of null exponents in our model is actually similar to that obtained in shell models of turbulence where the power law is seen at high drive values [28]. However, there are significant differences. First, we note that the shell model [28] cannot explain the crossover as it is only designed to explain the power law regime. Second, the maximum Lyapunov exponent is large for small viscosity parameter $\eta$, ie., $\lambda_{1} \propto \eta^{-1 / 2}$ in shell models [28] in contrast to near zero value in our model. It is also interesting to note that in our model propagating solutions arise in the power law regime of stress which comes as a surprise. As far as we are aware, this is the first situation, both from a experimental and theoretical angle, where propagating solutions are seen in a marginally stable situation.

Regarding the spatial features seen in the model, we stress that these features emerge purely due to dynamical reasons without any recourse to using the negative strain rate sensitivity feature as an input, as is the case in most models [50, 52, 53, 54]. Even the recently introduced poly-crystalline plasticity model which reproduces the crossover behavior also uses the negative SRS as an input [55]. The dynamical approach followed here clearly exposes how the slowing down of the plastic relaxation occurs due to a feed back mechanism of dislocation multiplication and applied strain rate as we reach the power law regime of stress drops. While the three different types of bands have features of the uncorrelated type $\mathrm{C}$, hopping type $\mathrm{B}$ and the propagating type $\mathrm{A}$ bands found in poly=crystalline materials, there is no element of poly-crystallinity in the model in its present form. In polycrystals, other types of coupling terms do arise which have also been modelled by diffusive type terms [1]. One way of including the presence of grain boundaries within the natural setting of the model is to recognize that crossslip will be hindered near the grain boundaries which also leads to a term similar to the present diffusive term. Such a term can account for the back stress arising from the incompatibility of grains. As the form of these terms are similar, the basic results are unlikely to change although one should expect a competition between the terms operating within a crystal and that at the grain boundaries.

From a purely dynamical point of view, this model should be of interest to the area of dynamical systems, as it appears to be the first fully dynamical model which exhibits a crossover from a chaotic to a power law regime, in the sense that our 
model is continuous space time model without any recourse to artificial thresholds as is done in coupled map lattices [56]. We note also that while the slow manifold subspace gives a method of visualizing the dislocations configurations, particularly in the scaling regime, the complementary subspace of the fast variable has helped us to obtain the band velocity in the same regime of strain rates. From the point of view of plastic instabilities, the present dynamical approach should be a promising direction for explaining many other patterns mentioned in the introduction [1].

Finally, as stated in the introduction, the PLC effect bears considerable similarity with many stick-slip systems and hence presents a way of understanding some of these systems. Here we make a few comments on the similarity of the present model for a possible adoption to the observed voltage fluctuations in charge density wave (CDW) compounds [11, 59]. Under the action of applied electric field, anomalously large voltage fluctuations are reported when the electric field is above the threshold value. This ohmic to non-ohmic transition in $K_{0.3} \mathrm{MoO}_{3}$ and $\mathrm{Rb}_{0.3} \mathrm{MoO}_{3}$, for instance [57], has not been adequately explained although the similarity with the PLC effect has been noted [11]. Lee and Rice [58] have suggested that phase dislocations of the CDW carry current at fields too low for the CDW to move as a whole. Indeed both fall in the category of pinning-depinning phenomenon. In the case of CDW, pinned at impurity/defect sites is unpinned due to the applied electric field. The threshold value of the electric field can be viewed as the onset of plastic flow of the charged phase dislocations [11]. These authors identify stress with voltage and strain with current and suggest that the total current is the sum of the ohmic part and that arising from charge density waves. This is corresponds to the elastic and plastic displacements competing to give rise to the PLC instability. We believe that this parallel can be taken further along the lines of our PLC model where one can identify the phase dislocations with mobile dislocations, the neutral defects of the CDW with dislocation dipoles, ie., the immobile, and the phase dislocations pinned at defects with the Cottrell type. Work along these lines is progress.

This work is supported by Department of Science and Technology, New Delhi, India.
[1] L.P. Kubin, C. Fressengeas and G. Ananthakrishna, in Collective Behaviour of Dislocations, edited by F.R.N. Nabarro and M.S. Deusbery, Dislocations in Solids Vol.11. P 101 (NorthHolland, Amsterdam, 2002).

[2] A detailed study of this effect is due to F. Le Chatelier, Rev. de Métall. 6, 914 (1909). However, the phenonmenon was first observed by Savart, F. Savart, Ann. Chim. Phys. Second series 65, 337 (1937).

[3] M. Zaiser and P. Hähner, Phys. Stat. Solidi B 199, 267 (1997).

[4] A. H. Cottrell, Dislocations and Plastic Flow in Crystals. (Clarendon Press, Oxford, 1953).

[5] A. Van den Beukel, Phys. Stat. Solidi A30, 197 (1975).

[6] L.P. Kubin, and Y. Estrin, Acta. Metall. 33, 397 (1985)

[7] P. Penning, Acta Metall. 20, 1169 (1972)

[8] B.N.J. Persson and E. Tosatti, Physics of Sliding Friction. (Kluwer Academic Publishers, Dordrecht, 1996).

[9] J.M. Carlson and J.S. Langer, Phys. Rev. Lett. 62, 2632 (1989); Phys. Rev. A. 40, 6470 (1989).

[10] D. Maugis and M. Barquins, Adhesion, edited by K.W. Allen, Vol. 12. (Elsevier, London, 1988).

[11] J. Dumas and D. Feinberg, Europhys. Lett. 2, 555 (1986).

[12] G. Ananthakrishna and M.C. Valsakumar, J. Phys. D 15, L171 (1982).

[13] S. Rajesh and G. Ananthakrishna, Phys. Rev. E. 61, 3664 (2000).

[14] G. Ananthakrishna and M.C. Valsakumar, Phys. Lett. A95, 69 (1983).

[15] G. Ananthakrishna et al., Scripta. Metall. 32, 1731 (1995).

[16] S.J. Noronha, et al., Int. Jl. of Bifurcation and Chaos 7, 2577 (1997).

[17] A. J. Lichtenberg and M. A. Libermann, Regular and Chaotic Dyanmics,(Springer-Verlag, New York, 1991).

[18] H.D.I. Abarbanel, Analysis of Observed Chaotic Data. (Springer-Verlag, New York, 1996).

[19] G. Ananthakrishna et al., Phys. Rev. E 605455 (1999).

[20] M.S. Bharathi, et al., Phys. Rev. Lett. 87, 165508 (2001).

[21] P. Bak, C. Tang and K. Wiesenfeld, Phys. Rev. Lett. 59, 381
(1987); Phys. Rev. A 38, 364 (1988).

[22] P. Bak, How Nature Works. (Springer - Verlag, New York, 1996).

[23] H.J. Jensen, Self-Organized Criticality. (Cambridge University Press, Cambridge, 1998).

[24] G. D'Anna and F. Nori, Phys. Rev. Lett. 85, 4096(2000); S. V. Franklin et al, F. Martens and M. Marder, Phys. Rev. E 62, 8195 (2000); Phys. Rev. Lett. 78, 4502 (1997).

[25] N. H. Packard, J. P. Crutchfield, J. D. Former and R. S. Shaw, Phys. Rev. Lett. 45, 712 (1980).

[26] See for instance F. Heslot, B. Castaing and A. Libchaber, Phys. Rev. A. 36, 5780 (1987).

[27] T. Bohr, M.H. Jensen, G. Paladin and A. Vulpiani, Dynamical Systems Approach to Tubulence, ( Cambridge,United Kingdom, 1997 ).

[28] M. Yamada and K. Ohkitani, J. Phys. Soc. Jpn. 56, 4210 (1987);M. Yamada and K. Ohkitani, Phys. Rev. Lett. 60, 983 (1988); Progr. Theor. Phys. 79, 1265 (1988); A. Cristani et al, Physica D, 76, 239 (1994) and the references therein.

[29] B. Gutenberg and C. F. Richter, Ann. Goefis. 9, 1 (1956).

[30] P. Diodati, F. Marchesoni and S. Piazza, Phys. Rev. Lett. 67, 2239 (1991).

[31] X. Che and H. Suhl, Phys. Rev. Lett. 64, 1670 (1990); K. L. Babcock and R. M. Westervelt, Phys. Rev. Lett. 64, 2168 (1990).

[32] M.S. Bharathi and G. Ananthakrishna, Europhys. Lett. 48, 1355 (2002).

[33] M.S. Bharathi and G. Ananthakrishna, Phys. Rev. E 67, 065104R (2003).

[34] M.V. Glazov et al Appl. Phys. A 64, 373 (1997).

[35] M. Zaiser et al, Comp. Mater. Science 5, 35 (1999).

[36] M. Bekele and G. Ananthakrishna, Phys. Rev. E 56, 6917 (1997).

[37] S. Rajesh and G. Ananthakrishna, Physica D 140, 193 (2000).

[38] A. Milik et al, Int. J. of Bifurcation and Chaos 8, 505 (1998).

[39] P. Grassberger and I. Procaccia, Physica D 9, 189 (1983).

[40] M. Ding, C. Grebogi, E. Ott, T. Sauer and J. A Yorke, Phys. 
Rev. Lett. 70, 3872 (1993)

[41] D. Broomhead and G. King, Physica. D20, 217 (1987).

[42] G. Benettin, L. Galgani, A. Giorgilli and J. M. Strelcyn, Mechanica, 15, 9 (1980).

[43] D. Ruelle, Comm. Math. Phys. 87, 287 (1982).

[44] A. Erzan and S. Sinha, Phys. Rev. Lett. 66, 2750 (1991).

[45] M. de Sousa Vieira and A.J. Lichtenberg, Phys. Rev. E. 53, 1441 (1996).

[46] B. Cessac, Ph. Blanchard and T. Kruger, Phys. Rev. E 64, 016133 (2001).

[47] M.S. Bharathi, S. Rajesh and G. Ananthakrishna, Scripta Mater. 48, 1355 (2003).

[48] G. Dee and J. S. Langer, Phys. Rev, Lett. 50, 383 (1983).

[49] W. van Saarloos, Phys. Rev. A 37, 211 (1988).

[50] P. Hähner, A. Ziegenbein, E. Rizzi and H. Neuhäuser, Phys. Rev. B 65, 134109 (2002).

[51] A. Korbel, J. Zasadzinski and Z. Siekluka, Acta Metall. 24, 921 (1976).

[52] P. G. Mc Cormick, and C. P. Ling. Acta Metall. Mater. 43,1069
(1995).

[53] S. Zhang, P. G. McCormick, Y. Estrin, Acta Mater. 49, 1087 (2001).

[54] M. Lebyodkin, L. Dunin-Barkowskii, Y. Bréchet, Y. Estrin, L. P. Kubin., Acta Mater. 48, 2529 (2000) and the references therein.

[55] S. Kok, M. S. Bharathi, A. J. Beaudoin, C. Fressengeas, G. Ananthakrishna, L. P. Kubin and M. Lybyodkin, Acta Materialia, 51, 3651 (2003).

[56] H. Chate and P. Manneville, Physic D 32, 409 (1988); S. Sinha and D. Biswas, Phys. Rev. lett. 71, 2010 (1993); S. Sinha, Int. J. Mod. Phys. B 9875 (1994); T. Bohr et al, Phys. Rev. Lett. 86, 5482 (2001).

[57] J. Dumas, C. Schlenker, J. Marcus and R. Buder, Phys. Rev. Lett. 50, 757 (1983), J. Dumas and C. Schlenker, Lecture Notes in Physics, Vol. 217, 439 (1985).

[58] P. A. Lee and T. M. Rice, Phys. Rev. B 19, 3970 (1979).

[59] G. Ananthakrishna, J. Indian Inst. 78, 165 (1998). 